\title{
Asymptotic analysis of state-to-state tetraatomic reactions using row-orthonormal hyperspherical coordinates
}

\author{
Ken Museth and Aron Kuppermann ${ }^{\text {a) }}$ \\ Artur Amos Noyes Laboratory of Chemical Physics, Division of Chemistry and Chemical Engineering, \\ California Institute of Technology, Pasadena, California 91125
}

(Received 11 June 2001; accepted 13 August 2001)

\begin{abstract}
The state-to-state asymptotic analysis of tetraatomic reactions is presented. It is assumed that the four-atom time-independent partial wave Schrödinger equation has been solved subject to the condition that in the limit of very compact geometries the wave function vanishes. These solutions are initially obtained in body-fixed row-orthonormal hyperspherical coordinates and transformed in the asymptotic arrangement channel regions of nuclear configuration space to Jacobi body-fixed coordinates. From the latter, compact explicit expressions for the reactance $(\mathbf{R})$ and scattering $(\mathbf{S})$ matrices, useful for accurate numerical calculations, are obtained. The different systems of coordinates used and their interrelations are given. The approach described is particularly well suited for implementation on massively parallel architectures and is appropriate for the calculation of benchmark-quality state-to-state integral and differential cross sections on currently available computers. (C) 2001 American Institute of Physics. [DOI: 10.1063/1.1408288]
\end{abstract}

\section{INTRODUCTION}

Let us consider the scattering between two molecules comprising a tetraatomic system. Once the motion of the center-of-mass (CM) of the system is removed, the corresponding scattering process takes place in a ninedimensional configuration space. Three of these nine degrees of freedom are taken to be external Euler angles that describe the overall rotation of the system around its $\mathrm{CM}$ in a laboratory-fixed frame. By means of a partial wave expansion involving the Wigner rotation functions ${ }^{1}$ of these angles one can easily and rigorously reduce the corresponding ninedimensional time-independent Schrödinger equation to a set of coupled six-dimensional partial differential equations in the six remaining internal coordinates, one set for each total angular momentum quantum number $J$. However, solving these equations constitutes a major numerical task which to this date has not been performed accurately enough to yield benchmark quality fully state-resolved state-to-state differential cross sections.

A scheme based on the use of row-orthonormal hyperspherical coordinates ${ }^{2}$ for the strong interaction region, of arrangement channel hyperspherical coordinates in the separated or nearly separated arrangement channel region (the weak interaction region), and of Jacobi coordinates in the asymptotic regions, makes such a benchmark-quality solution possible. We shall briefly discuss this scheme and the associated asymptotic analysis.

This paper is organized as follows: In Sec. II we give explicit definitions of the different coordinate systems employed in the different regions of configuration space, and derive some important expressions for the transformations between them. In Sec. III we discuss the asymptotic behavior

\footnotetext{
a) Author to whom correspondence should be addressed; electronic mail: aron@caltech.edu
}

of the physical solutions of the Schrödinger equation, and derive the body-fixed partial wave $\mathbf{R}$ - and $\mathbf{S}$-matrices. Finally, a summary is given in Sec. IV.

\section{THE HYPERSPHERICAL COORDINATE APPROACH}

Since the definition of symmetrized hyperspherical coordinates for three-dimensional tri-atomic reactions was introduced in $1975,{ }^{3}$ the hyperspherical coordinate approach has proven to offer a very convenient and effective numerical scheme for solving the time-independent Schrödinger equation for triatomic systems. ${ }^{4-32}$ Recently, Clary and coworkers have suggested an extension of this approach to tetraatomic systems involving different hyperspherical coordinates in different arrangement channel regions. ${ }^{33}$ In this method, super-completeness problems between basis sets in the different coordinates must be overcome. Alternatively, the availability of the Hamiltonian for tetraatomic systems in row-orthonormal hyperspherical coordinates, which are essentially invariant under arrangement channel transformations, and of massively parallel high performance computers should permit the calculation of state-to-state differential cross sections for a large number of tetraatomic reactions, including four center ones, for which two bonds are broken and two new bonds concurrently formed.

The hyperspherical coordinates for an $N$ atom system is composed of a set of three external angles describing the overall orientation of the system in space, a hyperradius which is a measure of the size of the system and $3 N-7$ internal hyperangles that describe the distances between the $N$ atoms. It is possible to define many different kinds of hyperspherical coordinates for any given system, but common to all is the hyperradius, $\rho$, which is kinematically invariant, i.e., is the same for all the system's arrangement channels. This is of great importance when studying systems of high dimensionality as it allows one to define a local basis 

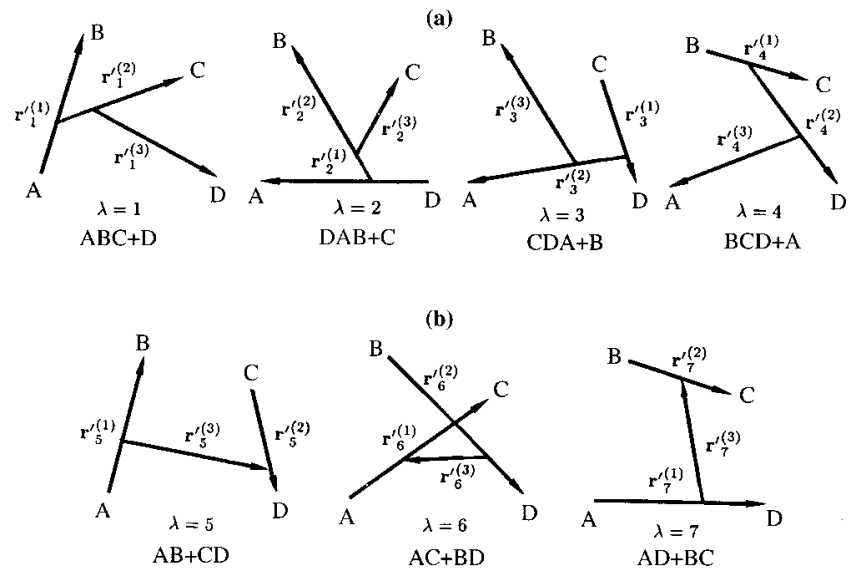

FIG. 1. Cluster arrangement channel coordinates: (a) atom-triatom clusters; (b) diatom-diatom clusters.

set for a constant $\rho$ which samples all of the different arrangement channels of interest. Thus in the hyperspherical coordinate approach, the system's wave function is expanded in local hyperspherical surface functions (LHSF) which depend on the internal hyperangles and parametrically on the hyperradius. These LHSF, for each value of $\rho$, form a complete orthonormal (and therefore linearly independent) basis set in these hyperangles, and as $\rho$ is varied they span all arrangement channel regions.

For a general tetraatomic system comprised of the distinct nuclei $A B C D$ we can form the following seven asymptotic arrangement channels: $A B C+D, D A B+C$, $C D A+B, B C D+A, A B+C D, A C+B D, A D+B C$. Of these, four are atom-triatom and three are diatom-diatom two-body arrangements. We assume that three-body channels (such as $A B+C+D$ ) as well as the fully dissociative channel $(A+B+C+D)$ are energetically closed. The hyperspherical formalism, including the asymptotic analysis, can be extended to include more than two-body channels, ${ }^{34}$ but this case will not be considered here. Illustrations of the corresponding seven cluster arrangement channel coordinates are shown in Fig. 3 of Ref. 35, and are repeated in Fig. 1 of the present paper for convenience. Throughout this paper we will use the symbol $\lambda=1,2, \ldots$ to label the different arrangement channels. In the case of identical nuclei we shall furthermore use the symbol $\Gamma$ to denote the irreducible representation of the corresponding permutation group. Generally speaking, we can have four different kinds of systems $A_{4}$, $A_{3} B, A_{2} B_{2}, A_{2} B C$, and $A B C D$ for which $\Gamma$, respectively, represents the irreducible representations of the permutation (or symmetric) groups $S_{4}, S_{3}, S_{2} \otimes S_{2}, S_{2}$ or $S_{1} \cdot{ }^{36}$ Thus arbitrary solutions of the time-independent Schrödinger equation will contain $\lambda$ and $\Gamma$ labels. As just mentioned one can define many different sets of hyperspherical coordinates for a given system, and in the rest of this section we shall briefly outline a calculation scheme which employs two such sets. Each of these sets of coordinates has distinct advantages in different regions of configuration space where the interactions between the atoms have different strengths. To facilitate the introduction of these different sets, we shall first assume that the full (nuclear) configuration space can be subdivided into a strong interaction region (for low values of $\rho$ ) and a weak interaction region (for large values of $\rho$ ). In addition, we will consider the asymptotic regions in which Jacobi coordinates will be used. This explicit description of the coordinates is necessary for the derivations described in the asymptotic analysis, which is eventually done in body-fixed Jacobi coordinates, also defined in this section. Finally, this section concludes by deriving an important relation between the principal axis of inertia Euler angles and the body-fixed Jacobi Euler angles.

\section{A. Coordinates in the strong interaction region}

In the strong interaction region we shall use roworthonormal hyperspherical coordinates. They and the corresponding Hamiltonian operator were presented in detail previously ${ }^{2}$ and we shall restrict ourself to outlining the properties of these coordinates needed for the rest of this paper.

For a given tetraatomic clustering scheme $\lambda$ we label by $\mathbf{r}_{\lambda}^{(i)^{\prime}}(i=1,2,3)$ the unscaled Jacobi vectors (see Fig. 1) and define the corresponding mass-scaled vectors $\mathbf{r}_{\lambda}^{(i)}$ by

$$
\mathbf{r}_{\lambda}^{(i)} \equiv\left(\mu_{\lambda}^{(i)} / \mu\right)^{1 / 2} \mathbf{r}_{\lambda}^{(i)^{\prime}}, \quad i=1,2,3
$$

where $\mu_{\lambda}^{(i)}$ and $\mu$ are the usual Delves reduced masses. ${ }^{37,38}$ From these mass-scaled Jacobi vectors we define a $3 \times 3$ Jacobi matrix $^{2}$ as

$$
\boldsymbol{\rho}_{\lambda}^{\mathrm{sf}} \equiv\left(\mathbf{r}_{\lambda}^{(1)} \mathbf{r}_{\lambda}^{(2)} \mathbf{r}_{\lambda}^{(3)}\right)=\left(\begin{array}{ccc}
x_{\lambda}^{(1)} & x_{\lambda}^{(2)} & x_{\lambda}^{(3)} \\
y_{\lambda}^{(1)} & y_{\lambda}^{(2)} & y_{\lambda}^{(3)} \\
z_{\lambda}^{(1)} & z_{\lambda}^{(2)} & z_{\lambda}^{(3)}
\end{array}\right),
$$

where the superscript "sf" denotes that the Jacobi vectors are defined in a space-fixed frame of reference, with $\left(x_{\lambda}^{(i)}, y_{\lambda}^{(i)}, z_{\lambda}^{(i)}\right)$ being the associated Cartesian coordinates of $r_{\lambda}^{(i)}(i=1,2,3)$. The ten row-orthonormal hyperspherical coordinates (ROHC) $\left\{\chi, a_{\lambda}^{\mathcal{I}}, b_{\lambda}^{\mathcal{I}}, c_{\lambda}^{\mathcal{I}}, \rho, \theta, \phi, \delta_{\lambda}^{(1)}, \delta_{\lambda}^{(2)}, \delta_{\lambda}^{(3)}\right\}$ are defined by the relation

$$
\boldsymbol{\rho}_{\lambda}^{\mathrm{sf}}=(-1)^{\chi} \widetilde{\mathbf{R}}\left(a_{\lambda}^{\mathcal{I}}, b_{\lambda}^{\mathcal{I}}, c_{\lambda}^{\mathcal{I}}\right) \rho \mathbf{N}(\theta, \phi) \widetilde{\mathbf{R}}\left(\delta_{\lambda}^{(1)}, \delta_{\lambda}^{(2)}, \delta_{\lambda}^{(3)}\right),
$$

where $\widetilde{\mathbf{R}}$ denotes the transposed of the usual proper rotation matrix

$$
\begin{aligned}
\mathbf{R}(a, b, c) \equiv & \left(\begin{array}{ccc}
\cos c & \sin c & 0 \\
-\sin c & \cos c & 0 \\
0 & 0 & 1
\end{array}\right) \\
& \times\left(\begin{array}{ccc}
\cos b & 0 & -\sin b \\
0 & 1 & 0 \\
\sin b & 0 & \cos b
\end{array}\right) \\
& \times\left(\begin{array}{ccc}
\cos a & \sin a & 0 \\
-\sin a & \cos a & 0 \\
0 & 0 & 1
\end{array}\right),
\end{aligned}
$$

and $\mathbf{N}$ is the simple diagonal matrix 


$$
\mathbf{N}(\theta, \phi)=\left(\begin{array}{ccc}
\sin \theta \cos \phi & 0 & 0 \\
0 & \sin \theta \sin \phi & 0 \\
0 & 0 & \cos \theta
\end{array}\right) .
$$

The chirality coordinate $\chi \equiv 0,1$ is defined by

$$
(-1)^{\chi}=\operatorname{sign}\left(\operatorname{det} \boldsymbol{\rho}_{\lambda}^{\text {sf }}\right) .
$$

$\rho$ is the usual hyperradius defined by $\rho^{2} \equiv \sum_{i=1}^{3}\left(r_{\lambda}^{(i)}\right)^{2}$ and $\mathbf{a}_{\lambda}^{\mathcal{I}} \equiv\left(a_{\lambda}^{\mathcal{I}}, b_{\lambda}^{\mathcal{I}}, c_{\lambda}^{\mathcal{I}}\right)$ denotes the external Euler angles which rotate the space-fixed frame of reference to the principal-axesof-inertia body-fixed frame, and are in the ranges $0 \leqslant a_{\lambda}^{\mathcal{I}}$, $c_{\lambda}^{\mathcal{I}}<2 \pi$ and $0 \leqslant b_{\lambda}^{\mathcal{I}} \leqslant \pi$. The two angles $0 \leqslant \theta \leqslant \arcsin [1 /(1$ $\left.\left.+\cos ^{2} \phi\right)\right]^{1 / 2}$ and $0 \leqslant \phi \leqslant \pi / 4$ determine the system's principal moments of inertia and finally $\delta_{\lambda} \equiv\left(\delta_{\lambda}^{(1)}, \delta_{\lambda}^{(2)}, \delta_{\lambda}^{(3)}\right)$, where $0 \leqslant \delta_{\lambda}^{(1)}, \delta_{\lambda}^{(3)}<\pi$ and $0 \leqslant \delta_{\lambda}^{(2)} \leqslant \pi$, are three internal hyperangles which describe the relative arrangements of the four atoms for a given set of those moments. Equation (3), together with these ranges, uniquely defines the ten ROHC. ${ }^{2}$

These hyperspherical coordinates have many advantageous features for the dynamics of the system, but for this discussion we shall only stress two important ones. First, four of the ten coordinates $(\chi, \rho, \theta, \phi)$ are arrangement-channel independent (i.e., are independent of $\lambda$ ). Second, the principal axes of inertia associated with $\left(a_{\lambda}^{\mathcal{I}}, b_{\lambda}^{\mathcal{I}}, c_{\lambda}^{\mathcal{I}}\right)$ have directions which are $\lambda$-independent; the senses of either two or none of these axis may change as $\lambda$ is changed. In addition, associated with the angles $\left(\delta_{\lambda}^{(1)}, \delta_{\lambda}^{(2)}, \delta_{\lambda}^{(3)}\right)$ there is a set of internal mathematical axis which have properties similar to those of the physical space principal axes of inertia, i.e., have directions which are $\lambda$-independent but senses such that two of them may change with $\lambda$. As a result, this set of roworthonormal coordinates is especially well suited for the description of the dynamics in the very strong interaction region. Furthermore, an analysis of the form of the corresponding Hamiltonian operator reveals that the permutation-symmetry of the system can be introduced very simply.

\section{B. Coordinates in the asymptotic region}

Let us now define the Jacobi coordinates in the asymptotic region. Using the mass-scaled Jacobi vectors $\left(\mathbf{r}_{\lambda}^{(1)}, \mathbf{r}_{\lambda}^{(2)}, \mathbf{r}_{\lambda}^{(3)}\right)$, given by Eq. (1), we can uniquely define the nine Jacobi coordinates $\left(r_{\lambda}^{(3)}, \theta_{\lambda}, \phi_{\lambda}, r_{\lambda}^{(2)}, \gamma_{\lambda}^{(2,3)}, \psi_{\lambda}, r_{\lambda}^{(1)}\right.$, $\left.\gamma_{\lambda}^{(1,3)}, \xi_{\lambda}\right)$ in the following way: $\left(r_{\lambda}^{(3)}, \theta_{\lambda}, \phi_{\lambda}\right)$ are the polar coordinates of $\mathbf{r}_{\lambda}^{(3)}$ in the space-fixed frame of reference $O x_{\lambda}^{\text {sf }} y_{\lambda}^{\text {sf }} z_{\lambda}^{\text {sf }},\left(r_{\lambda}^{(2)}, \gamma_{\lambda}^{(2,3)}, \psi_{\lambda}\right)$ are the polar coordinates of $\mathbf{r}_{\lambda}^{(2)}$ in the body-fixed frame $O x_{\lambda}^{\mathrm{bf}_{1}} y_{\lambda}^{\mathrm{bf}_{1}} z_{\lambda}^{\mathrm{bf}_{1}}$ which is obtained by the rotation of $O x_{\lambda}^{\mathrm{sf}} y_{\lambda}^{\mathrm{sf}} z_{\lambda}^{\text {sf }}$ by the Euler angles $\left(\phi_{\lambda}, \theta_{\lambda}, 0\right)$; and $\left(r_{\lambda}^{(1)}, \gamma_{\lambda}^{(1,3)}, \xi_{\lambda}\right)$ are the polar coordinates of $\mathbf{r}_{\lambda}^{(1)}$ in the bodyfixed frame $O x_{\lambda}^{\mathrm{bf}_{2}} y_{\lambda}^{\mathrm{bf}_{2}} z_{\lambda}^{\mathrm{bf}_{2}}$ which is obtained by rotation of $O x_{\lambda}^{\mathrm{bf}_{1}} y_{\lambda}^{\mathrm{bf}_{1}} z_{\lambda}^{\mathrm{bf}_{1}}$ by $\left(0,0, \psi_{\lambda}\right)$. These transformations are summarized in Table I. As a result, the $O z_{\lambda}^{\mathrm{bf}_{1}}$ and $O z_{\lambda}^{\mathrm{bf}_{2}}$ axis coincide and point along $\mathbf{r}_{\lambda}^{(3)}$, and $\mathbf{r}_{\lambda}^{(2)}$ lies is in the positive $O x_{\lambda}^{\mathrm{bf} z_{\lambda}} z_{\lambda}^{\mathrm{bf}_{2}}$ half-plane, with $O z_{\lambda}^{\mathrm{bf}_{1}} \equiv O z_{\lambda}^{\mathrm{bf}}$. For convenience we define the following compact notations for the corresponding Euler angles
TABLE I. Relation between Cartesian frames.

\begin{tabular}{lclll}
\hline \hline & $O x_{\lambda}^{\mathrm{sf}} y_{\lambda}^{\mathrm{sf}} z_{\lambda}^{\mathrm{sf}} \stackrel{\left(\phi_{\lambda}, \theta_{\lambda}, 0\right)}{\longrightarrow}$ & $O x_{\lambda}^{\mathrm{bf}_{1}} y_{\lambda}^{\mathrm{bf}_{1}} z_{\lambda}^{\mathrm{bf}_{1}} \stackrel{\left(0,0, \psi_{\lambda}\right)}{\longrightarrow}$ & $O x_{\lambda}^{\mathrm{bf}_{2}} y_{\lambda}^{\mathrm{bf}_{2}} z_{\lambda}^{\mathrm{bf}_{2}}$ \\
\hline $\mathbf{r}_{\lambda}^{(3)}$ & $\left(r_{\lambda}^{(3)}, \theta_{\lambda}, \phi_{\lambda}\right)$ & $\left(r_{\lambda}^{(3)}, 0,0\right)$ & & $\left(r_{\lambda}^{(3)}, 0,0\right)$ \\
$\mathbf{r}_{\lambda}^{(2)}$ & & $\left(r_{\lambda}^{(2)}, \gamma_{\lambda}^{(2,3)}, \psi_{\lambda}\right)$ & $\left(r_{\lambda}^{(2)}, \gamma_{\lambda}^{(2,3)}, 0\right)$ \\
$\mathbf{r}_{\lambda}^{(1)}$ & & & $\left(r_{\lambda}^{(1)}, \gamma_{\lambda}^{(1,3)}, \xi_{\lambda}\right)$ \\
\hline \hline
\end{tabular}

$$
\begin{aligned}
& \mathbf{a}_{\lambda}^{\mathrm{bf}_{1}}=\left(a_{\lambda}^{\mathrm{bf}_{1}}, b_{\lambda}^{\mathrm{bf}_{1}}, c_{\lambda}^{\mathrm{bf}_{1}}\right) \equiv\left(\phi_{\lambda}, \theta_{\lambda}, 0\right)=\left(\hat{\mathbf{r}}_{\lambda}^{(3)}, 0\right), \\
& \mathbf{a}_{\lambda}^{\mathrm{bf}_{2}}=\left(a_{\lambda}^{\mathrm{bf}_{2}}, b_{\lambda}^{\mathrm{bf}_{2}}, c_{\lambda}^{\mathrm{bf}_{2}}\right) \equiv\left(\phi_{\lambda}, \theta_{\lambda}, \omega_{\lambda}\right) .
\end{aligned}
$$

The angles $\gamma_{\lambda}^{(i, j)}$ between $r_{\lambda}^{(i)}$ and $r_{\lambda}^{(j)}$ are all in the 0 to $\pi$ range and are related by the simple expression

$$
\begin{aligned}
\cos \gamma_{\lambda}^{(1,2)}= & \cos \gamma_{\lambda}^{(1,3)} \cos \gamma_{\lambda}^{(2,3)} \\
& +\sin \gamma_{\lambda}^{(1,3)} \sin \gamma_{\lambda}^{(2,3)} \cos \xi_{\lambda},
\end{aligned}
$$

where $\xi_{\lambda}$ was defined in the last row of Table I and is in the range $[0,2 \pi]$. As a result, we can consider either the variables $\left(\gamma_{\lambda}^{(2,3)}, \gamma_{\lambda}^{(1,3)}, \xi_{\lambda}\right)$ or $\left(\gamma_{\lambda}^{(1,2)}, \gamma_{\lambda}^{(1,3)}, \gamma_{\lambda}^{(2,3)}, \chi\right)$ to describe the system. To go from $\gamma_{\lambda} \equiv\left(\gamma_{\lambda}^{(1,2)}, \gamma_{\lambda}^{(1,3)}, \gamma_{\lambda}^{(2,3)}\right)$ to $\left(\gamma_{\lambda}^{(2,3)}, \gamma_{\lambda}^{(1,3)}, \xi_{\lambda}\right)$, requires consideration of the chirality coordinate $\chi$, as discussed in Eq. (5.74) of Ref. 35. The (bodyfixed-2) Jacobi coordinates are, by definition, the ten coordinates

$$
\begin{aligned}
& \left(r_{\lambda}^{(1)}, r_{\lambda}^{(2)}, r_{\lambda}^{(3)}, \mathbf{a}_{\lambda}^{\mathrm{bf}_{2}}, \gamma_{\lambda}, \chi\right) \\
& \quad \equiv\left(r_{\lambda}^{(1)}, r_{\lambda}^{(2)}, r_{\lambda}^{(3)}, \phi_{\lambda}, \theta_{\lambda}, \psi_{\lambda}, \gamma_{\lambda}^{(1,2)}, \gamma_{\lambda}^{(1,3)}, \gamma_{\lambda}^{(2,3)}, \chi\right) .
\end{aligned}
$$

In these coordinates the Hamiltonian is independent of $\chi$ and has the simple form

$$
\begin{aligned}
\hat{H}_{\lambda}= & -\frac{\hbar^{2}}{2 \mu} \sum_{i=1}^{3}\left[\frac{1}{r_{\lambda}^{(i)}} \frac{\partial^{2}}{\left(\partial r_{\lambda}^{(i)}\right)^{2}} r_{\lambda}^{(i)}+\frac{\left(\hat{j}_{\lambda}^{(i)}\right)^{2}}{\left(r_{\lambda}^{(i)}\right)^{2}}\right]+V_{\lambda} \\
\equiv & -\frac{\hbar^{2}}{2 \mu}\left[\frac{1}{r_{\lambda}^{(3)}} \frac{\partial^{2}}{\left(\partial r_{\lambda}^{(3)}\right)^{2}} r_{\lambda}^{(3)}+\frac{1}{r_{\lambda}^{(2)}} \frac{\partial^{2}}{\left(\partial r_{\lambda}^{(2)}\right)^{2}} r_{\lambda}^{(2)}\right. \\
& \left.+\frac{1}{r_{\lambda}^{(1)}} \frac{\partial^{2}}{\left(\partial r_{\lambda}^{(1)}\right)^{2}} r_{\lambda}^{(1)}\right]+\frac{\hat{l}_{\mathrm{sf}}^{2}\left(\hat{\mathbf{r}}_{\lambda}^{(3)}\right)}{2 \mu\left(r_{\lambda}^{(3)}\right)^{2}}+\frac{\hat{j}_{\mathrm{bf}_{1}}^{2}\left(\hat{\mathbf{r}}_{\lambda}^{(2)}\right)}{2 \mu\left(r_{\lambda}^{(2)}\right)^{2}} \\
& +\frac{\hat{j}_{\mathrm{bf}_{2}}^{2}\left(\hat{\mathbf{r}}_{\lambda}^{(1)}\right)}{2 \mu\left(r_{\lambda}^{(1)}\right)^{2}}+V_{\lambda}\left(r_{\lambda}^{(1)}, r_{\lambda}^{(2)}, r_{\lambda}^{(3)}, \boldsymbol{\gamma}_{\lambda}\right),
\end{aligned}
$$

where $\hat{\mathbf{l}}_{\mathrm{sf}}\left(\hat{\mathbf{r}}_{\lambda}^{(3)}\right) \equiv \hat{\mathbf{j}}_{\lambda}^{(3)}$ is the space-fixed orbital angular momentum operator associated with $\mathbf{r}_{\lambda}^{(3)}, \hat{\mathbf{j}}_{\mathrm{bf}_{1}}\left(\hat{\mathbf{r}}_{\lambda}^{(2)}\right) \equiv \hat{\mathbf{j}}_{\lambda}^{(2)}$ is the body-fixed-1 angular momentum operator associated with $\mathbf{r}_{\lambda}^{(2)}$ and $\hat{\mathbf{j}}_{\mathrm{bf}_{2}}\left(\hat{\mathbf{r}}_{\lambda}^{(1)}\right) \equiv \hat{\mathbf{j}}_{\lambda}^{(1)}$ is the body-fixed-2 angular momentum operator associated with $\mathbf{r}_{\lambda}^{(1)}$, the angles involved being those given in the rightmost column of Table I. Note also that the potential energy, $V\left(r_{\lambda}^{(1)}, r_{\lambda}^{(2)}, r_{\lambda}^{(3)}, \gamma_{\lambda}\right)$, is neither a function of the three Euler angles $\mathbf{a}_{\lambda} \mathrm{bf}_{2}$, nor of the chirality variable $\chi$ but only of the intra-molecular coordinates. This of course stems from the fact that the intra-molecular forces are independent of the orientation of the tetraatomic system in space or of its chirality. The asymptotic form of the wave function is particularly simple in these coordinates, as shown in Sec. III B. 


\section{Coordinates in the weak interaction region}

The boundary between the strong and weak interaction regions is specified by a value $\rho_{b}$ of $\rho$. The criterion for determining $\rho_{b}$ is that the matrix elements of the interaction potential between all pairs of weak interaction LHSF belonging to different arrangement channels be sufficiently small (but not necessarily zero). In an actual calculation, $\rho_{b}$ should be treated as a convergence parameter.

In the weak interaction region a set of ten Delves hyperspherical coordinates are employed which are closely related to the Jacobi coordinates introduced in the previous section. ${ }^{35}$ They consist of three Euler angles (to be specified later), the chirality coordinate $\chi$, the three internal hyperangles $\gamma_{\lambda}$ defined after Eq. (8), the usual clustering-schemeindependent hyper-radius $\rho$, and the two internal hyperangles $\boldsymbol{\eta}_{\lambda}=\left(\eta_{\lambda}^{(1)}, \eta_{\lambda}^{(2)}\right)$ defined by the relations

$$
\begin{aligned}
& r_{\lambda}^{(1)}=\rho \sin \eta_{\lambda}^{(1)} \sin \eta_{\lambda}^{(2)}, \\
& r_{\lambda}^{(2)}=\rho \cos \eta_{\lambda}^{(1)} \sin \eta_{\lambda}^{(2)}, \\
& r_{\lambda}^{(3)}=\rho \cos \eta_{\lambda}^{(2)},
\end{aligned}
$$

where the ranges of the angles are $0 \leqslant \eta_{\lambda}^{(1)}, \eta_{\lambda}^{(2)} \leqslant \pi / 2$. Thus in going from the asymptotic region, discussed in the previous subsection, to the weak interaction region, we use the same geometrical angular coordinates but the Jacobi coordinates $\left(r_{\lambda}^{(1)}, r_{\lambda}^{(2)}, r_{\lambda}^{(3)}\right)$ are replaced by $\left(\rho, \eta_{\lambda}^{(1)}, \eta_{\lambda}^{(2)}\right)$. However, it is very important to stress that we have a freedom of choice for the three external (Euler) hyperangles; even though the $\mathbf{a}_{\lambda}^{\mathrm{bf}_{2}}$ of Eq. (7b) uniquely defined the Euler angles for the Jacobi coordinates-which in turn defined a subset of the Delves hyperspherical coordinates, Eq. (11) — the Jacobi distances $\left(r_{\lambda}^{(1)}, r_{\lambda}^{(2)}, r_{\lambda}^{(3)}\right)$ and the corresponding angles $\gamma_{\lambda}$ between them are totally independent of any frame of reference. Consequently we shall choose the external hyperangles which lead to the overall most convenient numerical scheme. From the previous subsections we know that on either "side" of the weak interaction region different frames of reference are employed; in the strong interaction region we use the principal-axis-of-inertia frame of reference defined by $\mathbf{a}_{\lambda}^{\mathcal{I}}$, whereas in the asymptotic region we use the body-fixed-2 frame specified by $\mathbf{a}_{\lambda}^{\mathrm{bf}_{2}}$. A transformation between one of these frames and the other will have to be done either at the boundary between the strong and weak interaction regions, or between the latter and the asymptotic region. Hence, it would be convenient to use one of these two sets of Euler angles in the intermediate weak interaction region. Since the $\mathbf{a}_{\lambda}^{\mathcal{I}}$ angles asymptotically approach the $\mathbf{a}_{\lambda}^{\mathrm{bf}_{2}}$ angles, it is numerically most convenient to employ the principal-axes-ofinertia frame of reference in the strong and in the weak interaction regions. This leads to the following choice for the Delves hyperspherical coordinates in the weak interaction region:

$$
\begin{aligned}
& \left(\rho, \mathbf{a}_{\lambda}^{\mathcal{I}}, \boldsymbol{\eta}_{\lambda}, \boldsymbol{\gamma}_{\lambda}, \chi\right) \\
& \quad \equiv\left(\rho, a_{\lambda}^{\mathcal{I}}, b_{\lambda}^{\mathcal{I}}, c_{\lambda}^{\mathcal{I}}, \eta_{\lambda}^{(1)}, \eta_{\lambda}^{(2)}, \gamma_{\lambda}^{(1,2)}, \gamma_{\lambda}^{(1,3)}, \gamma_{\lambda}^{(2,3)}, \chi\right)
\end{aligned}
$$

Because of the orthogonal nature of the transformation defined by Eq. (11), it can easily be shown that under that transformation Eq. (10) becomes

$$
\begin{aligned}
\hat{H}_{\lambda} \equiv & -\frac{\hbar^{2}}{2 \mu \rho^{8}} \frac{\partial}{\partial \rho} \rho^{8} \frac{\partial}{\partial \rho}+\frac{\mathcal{L}_{1}^{2}\left(\boldsymbol{\eta}_{\lambda}\right)+\mathcal{L}_{2}^{2}\left(\boldsymbol{\eta}_{\lambda}\right)}{2 \mu \rho^{2}} \\
& +\frac{\hat{l}_{\mathrm{sf}}^{2}\left(\hat{\mathbf{r}}_{\lambda}^{(3)}\right)}{2 \mu\left(r_{\lambda}^{(3)}\right)^{2}}+\frac{\hat{j}_{\mathrm{bf}_{1}}^{2}\left(\hat{\mathbf{r}}_{\lambda}^{(2)}\right)}{2 \mu\left(r_{\lambda}^{(2)}\right)^{2}}+\frac{\hat{j}_{\mathrm{bf}_{2}}^{2}\left(\hat{\mathbf{r}}_{\lambda}^{(1)}\right)}{2 \mu\left(r_{\lambda}^{(1)}\right)^{2}} \\
& +V_{\lambda}\left(\rho, \eta_{\lambda}^{(1)}, \eta_{\lambda}^{(2)}, \gamma_{\lambda}^{(1,2)}, \gamma_{\lambda}^{(1,3)}, \gamma_{\lambda}^{(2,3)}\right),
\end{aligned}
$$

where hyperangular momentum operators $\hat{\mathcal{L}}_{1}^{2}$ and $\hat{\mathcal{L}}_{2}^{2}$ are defined by

$$
\begin{aligned}
\hat{\mathcal{L}}_{1}^{2}\left(\boldsymbol{\eta}_{\lambda}\right) \equiv & -\hbar^{2}\left(\frac{2}{\sin \eta_{\lambda}^{(2)} \sin 2 \eta_{\lambda}^{(1)}}\right)^{2} \frac{\partial}{\partial\left(2 \eta_{\lambda}^{(1)}\right)} \\
& \times\left(\sin 2 \eta_{\lambda}^{(1)}\right)^{2} \frac{\partial}{\partial\left(2 \eta_{\lambda}^{(1)}\right)}
\end{aligned}
$$

$$
\begin{aligned}
\hat{\mathcal{L}}_{2}^{2}\left(\boldsymbol{\eta}_{\lambda}\right) \equiv & -\hbar^{2} \frac{1}{\left(\sin \eta_{\lambda}^{(2)}\right)^{5}\left(\cos \eta_{\lambda}^{(2)}\right)^{2}} \frac{\partial}{\partial\left(\eta_{\lambda}^{(2)}\right)} \\
& \times\left(\sin \eta_{\lambda}^{(2)}\right)^{5}\left(\cos \eta_{\lambda}^{(2)}\right)^{2} \frac{\partial}{\partial\left(\eta_{\lambda}^{(2)}\right)} .
\end{aligned}
$$

In Eq. (13), $\hat{\mathbf{j}}_{\mathrm{bf}_{1}}$ and $\hat{\mathbf{j}}_{\mathrm{bf}_{2}}$ are still referring to the $\mathrm{bf}_{1}$ and $\mathrm{bf}_{2}$ axes, respectively. The transformation of principal axis of inertia axes to the $\mathrm{bf}_{2}$ axes and their associates Euler angles is the topic of the next subsection.

\section{Transformation between the Euler angles}

As the asymptotic analysis will be conducted in the body-fixed-2 frame, defined in Sec. II B, and the scattering wave functions in the adjacent weak interaction region is expressed in the principal-axis-of-inertia frame, we need to establish the relation between the corresponding Euler angles. For reasons that will be clarified in the actual projection of the wave function onto its asymptotic form, it is desirable to express this frame transformation in terms of the (internal) Delves hyperspherical coordinates. This subsection outlines the derivation of such a relation, but for economy of space most of the lengthy details and explicit expressions have been left out. We emphasize, however, that all of the omitted derivations involve straightforward (but tedious) algebraic manipulations.

In terms of the $3 \times 3$ Jacobi matrix notation introduced in Sec. II A, we can conveniently express the relation between the three frames involved as

$$
\boldsymbol{\rho}_{\lambda}^{\mathrm{sf}}=\widetilde{\mathbf{R}}\left(\mathbf{a}_{\lambda}^{\mathcal{I}}\right) \boldsymbol{\rho}_{\lambda}^{\mathcal{I}}=\widetilde{\mathbf{R}}\left(\mathbf{a}_{\lambda}^{\mathrm{bf}_{2}}\right) \boldsymbol{\rho}_{\lambda}^{\mathrm{bf} f_{2}},
$$

where $\boldsymbol{\rho}_{\lambda}^{\text {sf }}$ denotes the Jacobi matrix in space-fixed coordinates, defined in Eq. (2). $\boldsymbol{\rho}_{\lambda}^{\mathcal{I}}$ and $\boldsymbol{\rho}_{\lambda}^{\mathrm{bf}_{2}}$ are the corresponding Jacobi matrices expressed respectively in the principal-axisof-inertia and the body-fixed-2 frames of references. By definition, 


$$
\boldsymbol{\rho}_{\lambda}^{\mathcal{I}}=(-1)^{\chi} \rho \mathbf{N}(\theta, \phi) \tilde{\mathbf{R}}\left(\boldsymbol{\delta}_{\lambda}\right) .
$$

From the definitions of the body-fixed-2 frame, schemati- cally outlined in Table I, and the transformations in Eq. (11) we furthermore have the following expression for the bodyfixed-2 Jacobi matrix expressed in Delves hyperspherical coordinates

$$
\boldsymbol{\rho}_{\lambda}^{\mathrm{bf}_{2}}=\rho\left(\begin{array}{ccc}
\sin \eta_{\lambda}^{(1)} \sin \eta_{\lambda}^{(2)} \sin \gamma_{\lambda}^{(1,3)} \cos \xi_{\lambda} & \cos \eta_{\lambda}^{(1)} \sin \eta_{\lambda}^{(2)} \sin \gamma_{\lambda}^{(2,3)} & 0 \\
\sin \eta_{\lambda}^{(1)} \sin \eta_{\lambda}^{(2)} \sin \gamma_{\lambda}^{(1,3)} \sin \xi_{\lambda} & 0 & 0 \\
\sin \eta_{\lambda}^{(1)} \sin \eta_{\lambda}^{(2)} \cos \gamma_{\lambda}^{(1,3)} & \cos \eta_{\lambda}^{(1)} \sin \eta_{\lambda}^{(2)} \cos \gamma_{\lambda}^{(2,3)} & \cos \eta_{\lambda}^{(2)}
\end{array}\right)
$$

Left multiplying Eq. (15) by $\mathbf{R}\left(\mathbf{a}_{\lambda}^{\mathrm{bf}_{2}}\right)$ we obtain

$$
\boldsymbol{\rho}_{\lambda}^{\mathrm{bf}_{2}}=\mathbf{R}\left(\mathbf{a}_{\lambda}^{\mathrm{bf}_{2}}\right) \tilde{\mathbf{R}}\left(\mathbf{a}_{\lambda}^{\mathcal{I}}\right) \boldsymbol{\rho}_{\lambda}^{\mathcal{I}} \equiv \mathbf{R}\left(\boldsymbol{\alpha}_{\lambda}\right) \boldsymbol{\rho}_{\lambda}^{\mathcal{I}},
$$

where we have introduced the three angles $\boldsymbol{\alpha}_{\lambda}$ which by definition rotate the principal-axis-of-inertia frame into the bodyfixed-2 frame. Right multiplying this expression by the inverse of $\boldsymbol{\rho}_{\lambda}^{\mathcal{I}}$ (which is nonsingular for nonplanar configurations) we arrive at the following expression:

$$
\mathbf{R}\left(\boldsymbol{\alpha}_{\lambda}\right)=\boldsymbol{\rho}_{\lambda}^{\mathrm{bf}_{2}}\left[\boldsymbol{\rho}_{\lambda}^{\mathcal{I}}\right]^{-1}=(-1)^{\chi} \boldsymbol{\rho}_{\lambda}^{\mathrm{bf}_{2}} \mathbf{R}\left(\boldsymbol{\delta}_{\lambda}\right)[\mathbf{N}(\theta, \phi)]^{-1}
$$

which relates the 3 angles $\boldsymbol{\alpha}_{\lambda}$ to the 11 internal coordinates $\chi, \boldsymbol{\eta}_{\lambda}, \boldsymbol{\gamma}_{\lambda}, \boldsymbol{\delta}_{\lambda}, \theta$ and $\phi$ ( $\rho$ cancels out). First we note that the angles $\boldsymbol{\alpha}_{\lambda}$ are indeed independent of the Euler angles $\mathbf{a}_{\lambda}^{\mathcal{I}}$ and $\mathbf{a}_{\lambda}^{\mathrm{bf}_{2}}$. Second, we note that the five internal row-orthonormal hyperangles $\boldsymbol{\delta}_{\boldsymbol{\lambda}}, \theta$, and $\phi$ can be expressed in terms of the five Delves hyperangles $\boldsymbol{\eta}_{\lambda}$ and $\boldsymbol{\gamma}_{\lambda}$. Indeed, by leftmultiplying Eq. (15) by its transpose, we get

$$
\tilde{\boldsymbol{\rho}}_{\lambda}^{\mathrm{sf}} \boldsymbol{\rho}_{\lambda}^{\mathrm{sf}}=\tilde{\boldsymbol{\rho}}_{\lambda}^{\mathrm{bf}_{2}} \boldsymbol{\rho}_{\lambda}^{\mathrm{bf} f_{2}}=\tilde{\boldsymbol{\rho}}_{\lambda}^{\mathcal{I}} \boldsymbol{\rho}_{\lambda}^{\mathcal{I}},
$$

where the third term, and therefore the first and second also, is independent of $\chi$. Substituting Eq. (16) and Eq. (17) into Eq. (20) makes it clear that one can derive closed expressions for $\boldsymbol{\delta}_{\lambda}, \theta$, and $\phi$ in terms of $\boldsymbol{\eta}_{\lambda}$ and $\boldsymbol{\gamma}_{\lambda}$ which can in turn be used to eliminate the former from Eq. (19). These derivations are straightforward but very lengthy and throughout the remaining of this paper we shall simply note that $\boldsymbol{\alpha}_{\lambda}$ can be expressed exclusively in terms of $\boldsymbol{\eta}_{\lambda}$ and $\boldsymbol{\gamma}_{\lambda}$ and we will write it as $\boldsymbol{\alpha}_{\lambda}\left(\boldsymbol{\eta}_{\lambda}, \boldsymbol{\gamma}_{\lambda}\right)$.

As a last auxiliary expression let us note that from the relation between the three $\mathbf{R}$ matrices in Eq. (18) the corresponding Wigner rotation matrices ${ }^{1}$ satisfy the relation

$$
\mathbf{D}^{J}\left(\mathbf{a}_{\lambda}^{\mathrm{bf}_{2}}\right)=\mathbf{D}^{J}\left(\mathbf{a}_{\lambda}^{\mathcal{I}}\right) \mathbf{D}^{J}\left(\boldsymbol{\alpha}_{\lambda}\right) .
$$

Inverting this matrix expression and rewriting it in terms of the individual matrix elements we arrive at the final important expression

$$
D_{M \Omega}^{J}\left(\mathbf{a}_{\lambda}^{\mathcal{I}}\right)=\sum_{K} D_{M K}^{J}\left(\mathbf{a}_{\lambda}^{\mathrm{bf}_{2}}\right) D_{\Omega K}^{J^{*}}\left(\boldsymbol{\alpha}_{\lambda}\left(\boldsymbol{\eta}_{\lambda}, \boldsymbol{\gamma}_{\lambda}\right)\right) .
$$

This concludes the prelude to the explicit asymptotic analysis to be presented in the next section.

\section{THE R,S ASYMPTOTIC ANALYSIS}

As mentioned in the Introduction, a tetraatomic collisional system with a total energy below that required for the system to dissociate into three or four bodies involves asymptotic arrangement channels that can be characterized either as atom-triatom or diatom-diatom channels. The wave functions in these two kinds of asymptotic channels are very different from each other and hence we shall treat them separately in the otherwise general tetraatomic asymptotic analysis to follow. However, to save space, we will, whenever possible, adopt a common notation for the two cases. As a result, notational conventions are especially important for this analysis.

The general asymptotic form of a time-independent tetraatomic physical scattering wave function $\Psi_{\text {phys }}^{\lambda^{\prime} n_{\lambda^{\prime}}^{\prime}}$ is

$$
\begin{aligned}
\Psi_{\text {phys }}^{\lambda^{\prime} n_{\lambda^{\prime}}^{\prime}} \sim \exp \left[i k_{\lambda{ }^{\prime} \epsilon_{\lambda^{\prime}}}, r_{\lambda^{\prime}}\right. & (3) \cos \theta_{\lambda^{\prime}} \Phi_{\lambda^{\prime} n_{\lambda^{\prime}}^{\prime}}\left(\mathbf{r}_{\lambda^{\prime}}^{(1)}, \mathbf{r}_{\lambda^{\prime}}^{(2)}\right) \\
& +\sum_{\lambda n_{\lambda}} f_{\lambda n_{\lambda}}^{\lambda^{\prime} n_{\lambda^{\prime}}}\left(\hat{\mathbf{r}}_{\lambda}^{(3)}\right) \frac{e^{i k_{\lambda \epsilon_{\lambda}{ }_{\lambda}} r_{\lambda}^{(3)}}}{r_{\lambda}^{(3)}} \Phi_{\lambda n_{\lambda}}\left(\mathbf{r}_{\lambda}^{(1)}, \mathbf{r}_{\lambda}^{(2)}\right)
\end{aligned}
$$

which corresponds to the collision of two isolated reactants $\left\{\lambda_{1}^{\prime}, \lambda_{2}^{\prime}\right\} \equiv \lambda^{\prime}$ to form the two products $\left\{\lambda_{1}, \lambda_{2}\right\} \equiv \lambda$. In this expression $\Phi_{\lambda n_{\lambda}}, \Phi_{\lambda^{\prime} n_{\lambda^{\prime}}^{\prime}}$ and $f_{\lambda n_{\lambda}}^{\lambda^{\prime} n_{\lambda^{\prime}}^{\prime}}$ denote, respectively, the product and reactant wave functions and the corresponding scattering amplitudes which depend on the angles $\hat{\mathbf{r}}_{\lambda}^{(3)}$ $\equiv\left(\theta_{\lambda}, \phi_{\lambda}\right)$ defined in Table I. The far asymptotic behavior of the physical wave function [expressed as the symbol $\sim$ in Eq. (23)] is composed of two distinct parts: the incident part which is the product of an incoming plane wave (arbitrarily assigned to be traveling in the space-fixed $z$-direction) and the reactant wave function, and the scattered part which is a sum over all possible product state wave functions multiplied by corresponding outgoing spherical waves and weighted by the associated scattering amplitudes. When $\lambda$ labels a diatom-diatom or an atom-triatom arrangement channel, $\Phi_{\lambda n_{\lambda}}\left(\mathbf{r}_{\lambda}^{(1)}, \mathbf{r}_{\lambda}^{(2)}\right)$ is, respectively, the product of the two diatomic wave functions or a triatomic wave function. The corresponding quantum numbers $\lambda n_{\lambda}$ for these two cases are, respectively, defined by the following compact notations: 
two diatoms:

$$
\begin{aligned}
\lambda n_{\lambda} & \equiv\left\{\lambda_{1}, \lambda_{2}, v_{\lambda}^{(1)}, v_{\lambda}^{(2)}, j_{\lambda}^{(1)}, j_{\lambda}^{(2)}, m_{\lambda}^{(1)}, m_{\lambda}^{(2)}\right\} \\
& =\left\{\lambda \epsilon_{\lambda}, m_{\lambda}^{(1)}, m_{\lambda}^{(2)}\right\},
\end{aligned}
$$

atom-triatom:

$$
\begin{aligned}
\lambda n_{\lambda} & \equiv\left\{\lambda_{1}, \lambda_{2}, \mathbf{v}_{\lambda}^{(1)}, j_{\lambda}^{(1)}, m_{\lambda}^{(1)}\right\} \\
& =\left\{\lambda \epsilon_{\lambda}, m_{\lambda}^{(1)}\right\} .
\end{aligned}
$$

We have, for convenience, introduced the subset of quantum numbers $\lambda \epsilon_{\lambda}$ on which the asymptotic molecules internal energies depend. Similar definitions are used for the two cases of reactants arrangement channels $\lambda^{\prime}$. These quantum numbers are associated with internal vibration $\left(v_{\lambda}^{(i)}\right)$ and rotation $\left(j_{\lambda}^{(i)}, m_{\lambda}^{(i)}\right)$ degrees of freedom. Thus $v_{\lambda}^{(1)}$ and $v_{\lambda}^{(2)}$ refer to the vibrational quantum number of each of the two diatomic fragments whereas the set $\mathbf{v}_{\lambda}^{(1)}$ is associated with the three vibrational degrees of freedom for a triatom. Similarly, $j_{\lambda}^{(1)}$ and $j_{\lambda}^{(2)}$ in Eq. (24a) correspond to the angular momentum of each of the two diatoms, and $j_{\lambda}^{(1)}$ in Eq. (24b) is the total angular momentum quantum number of the triatom (no spatial nuclear motion angular momentum is of course defined for an isolated atom). For the reactants the corresponding quantization axis for the $z$-component of the angular momenta, $m_{\lambda^{\prime}}^{\prime(i)}$, is chosen to be the direction of the incident relative velocity, i.e., the space-fixed $z$-axis. As was done for triatomic systems, ${ }^{39}$ we chose for the quantization axis of $j_{\lambda}^{(i)}$ the product Jacobi body-fixed-2 axis, $O z_{\lambda}{ }^{\mathrm{bf}_{2}}$ (see Table I). This is called a helicity representation and leads to the most compact and computationally convenient expressions for the state-to-state differential and integral cross sections. It should be emphasized that the sum over product states in the last term of Eq. (23) includes (in principle) all possible states, i.e., both open and closed ones. This ensures the mathematical completeness of the expansion, and can be understood when realizing that asymptotically closed states might well be energetically accessible in the inner parts of the configuration space.

Whereas it might seem reasonable from the discussions above to attempt to explicitly look for solutions of the Schrödinger equation which satisfy Eq. (23), it should be stressed that this type of approach is not usually numerically convenient as it can in fact only be done by some kind of "trial and error" recursive scheme. Thus instead of directly solving the Schrödinger equation subject to the physical boundary conditions, in order to obtain $f_{\lambda n_{\lambda}{ }^{\prime} n_{\lambda^{\prime}}^{\prime}}$, we use the standard ' $\mathbf{R}, \mathbf{S}$ matrix" approach, ${ }^{40,41}$ where one first solves the Schrödinger equation for general mathematical solutions (i.e., without imposing the physical asymptotic conditions). By a subsequent asymptotic analysis of these mathematical solutions the so-called R-matrix is obtained. Whereas the nonphysical solutions themselves are not unique, the R-matrix for a given partial $J$, parity $\Pi$ (with respect to inversion through the system's center of mass), irreducible representation $\Gamma$ of the permutation group of identical atoms, and energy $E$, is indeed unique. The partial wave $\mathbf{S}$-matrix is then obtained in a simple manner from the corresponding $\mathbf{R}$-matrix, and the physical scattering amplitudes, $f_{\lambda n_{\lambda}}^{\lambda^{\prime} n_{\lambda^{\prime}}^{\prime}}$, can next be calculated directly from partial wave sums over the corresponding S-matrices.

Thus the key feature of the $\mathbf{R}, \mathbf{S}$-matrix approach is to decouple the mathematical and physical parts of the scattering process (i.e., the solution of the Schrödinger equation and imposition of the asymptotic conditions) in such a way that the proper physical solutions are expressed as linear combinations of an arbitrary complete set of linearly independent mathematical solutions through the introduction of the $\mathbf{R}$ - and $\mathbf{S}$-matrices. In the following we outline the steps involved in computing these $\mathbf{R}$ - and $\mathbf{S}$-matrices from the mathematical solutions of the Schrödinger equation.

\section{A. Definition of the reactance and scattering matrices}

Let the wave functions $\Psi_{\lambda^{\prime} b_{\lambda^{\prime}}^{\prime}}^{J M \Pi \Gamma}$ constitute a complete set of simultaneous solutions of the following eigenvalueeigenfunction equations

$$
\begin{aligned}
& \hat{H} \Psi_{\lambda^{\prime} b_{\lambda^{\prime}}^{\prime}}^{J M \Pi \Gamma}=E \Psi_{\lambda^{\prime} b_{\lambda^{\prime}}^{\prime}}^{J M \Pi \Gamma}, \\
& \hat{J}^{2} \Psi_{\lambda^{\prime} b_{\lambda^{\prime}}^{\prime}}^{J M \Pi \Gamma}=J(J+1) \hbar^{2} \Psi_{\lambda^{\prime} b_{\lambda^{\prime}}^{\prime}}^{J M \Pi \Gamma}, \\
& \hat{J}_{\mathrm{sf}_{z}} \Psi_{\lambda^{\prime} b_{\lambda^{\prime}}^{\prime}}^{J M \Pi \Gamma}=M \hbar \Psi_{\lambda^{\prime} b_{\lambda^{\prime}}^{\prime}}^{J M \Pi \Gamma}, \\
& \hat{I} \Psi_{\lambda^{\prime} b_{\lambda^{\prime}}^{\prime}}^{J M \Pi \Gamma}=(-1)^{\Pi} \Psi_{\lambda^{\prime} b_{\lambda^{\prime}}^{\prime}}^{J M \Pi \Gamma},
\end{aligned}
$$

where $\hat{H}, \hat{J}^{2}, \hat{J}_{\text {sf }_{z}}$, and $\hat{I}$ are, respectively, the system's nuclear motion Hamiltonian, the square of its total angular momentum operator $\hat{\mathbf{J}}$, the space-fixed $z$ component of $\hat{\mathbf{J}}$, and the operator $\hat{I}$ which inverts the system through its center of mass. In addition, $E, J(J+1) \hbar^{2}, M \hbar$, and $(-1)^{\Pi}$ are the corresponding eigenvalues. These wave functions are required furthermore to transform according to the irreducible representations $\Gamma$ of the permutation group of identical atoms of the tetraatomic system. Such a set of simultaneous eigenfunctions does indeed exist since the corresponding operators commute. Finally, the subscript $\lambda^{\prime} b_{\lambda^{\prime}}^{\prime}$ labels the different linearly independent solutions and spans the same range as the indices defined by Eqs. (33) and (35) and associated with the isolated molecular products. These solutions are of the "mathematical" type, i.e., are not required to satisfy the physical asymptotic conditions of Eq. (23). We assume that this set of solutions has been computed by the hyperspherical coordinate approach as follows. We start in the strong interaction region at a sufficiently small hyperradius for the wave function to be close to zero, and expand the wave function in LHSFs expressed in the row-orthonormal hyperspherical coordinates. The coefficients of this expansion depend on $\rho$ only, and satisfy a set of coupled second order differential equations. These are propagated outwards for increasing $\rho$, until the boundary with the weak interaction region is reached. We then switch to the weak interaction Delves hyperspherical coordinates and surface functions and continue 
propagating in the $\rho$ variable until the boundary with the asymptotic region is reached. In the weak interaction region these solutions can be expressed as

$$
\begin{aligned}
\Psi_{\lambda^{\prime} b_{\lambda^{\prime}}^{\prime}}^{J M \Pi \Gamma}= & \rho^{-4} \sum_{\lambda b_{\lambda}} C_{\lambda b_{\lambda}}^{J \Pi \Gamma \lambda^{\prime} b_{\lambda^{\prime}}^{\prime}}(\rho ; \bar{\rho}) D_{M \Omega_{\lambda}}^{J}\left(\mathbf{a}_{\lambda}^{\mathcal{I}}\right) \\
& \times \Phi_{J \Pi \Gamma \lambda b_{\lambda}}^{\mathrm{LHSF}}\left(\boldsymbol{\eta}_{\lambda}, \boldsymbol{\gamma}_{\lambda} ; \overline{\boldsymbol{\rho}}\right) .
\end{aligned}
$$

In this expansion, $D_{M \Omega_{\lambda}}^{J}$ are the usual Wigner rotation matrices, ${ }^{1} \Phi_{J \Pi \Gamma \lambda b_{\lambda}}^{\mathrm{LHSF}}$ are the LHSF (displaying a parametric dependence on the hyperradius $\bar{\rho}$ ), and $C_{\lambda b_{\lambda}}^{J \Pi \Gamma \lambda^{\prime} b_{\lambda^{\prime}}^{\prime}}$ are $\rho$-dependent coefficients determined by the solution of Eq. (25a). ( $\rho^{-4}$ is a convenient multiplication factor which eliminates the first derivative with respect to $\rho$ contained in the hyperradial kinetic energy operator.) More specifically, the $\Phi_{J \Pi \Gamma \lambda b_{\lambda}}^{\mathrm{LHSF}}$ are defined as eigenfunctions of some conveniently chosen weak-interaction reference Hamiltonian that contains differential operators in the coordinates $\boldsymbol{\eta}_{\lambda}$ and $\boldsymbol{\gamma}_{\lambda}$ but not $\bar{\rho}{ }^{4,9}$ The explicit definition of this LHSF reference Hamiltonian is outside the scope of this paper and we just noticed that it is obtained from Eq. (13) after the analytical elimination of the Euler angles, $\mathbf{a}_{\lambda}^{\mathcal{I}}$, by means of a partialwave expansion of in $J$ and the freezing of the hyperradius $\rho$. Because of the rotational invariance of space the $C_{\lambda b_{\lambda}}^{J \Pi \Gamma \lambda^{\prime} b_{\lambda^{\prime}}^{\prime}}$ and $\Phi_{J \Pi \Gamma \lambda b_{\lambda}}^{\mathrm{LHSF}}$ are independent of $M$, and the entire $M$-dependence of $\Psi_{\lambda^{\prime} b^{\prime},}^{J M \Pi \Gamma}$ is expressed by the $D_{M \Omega_{\lambda}}^{J}$.

The $\mathbf{R}^{J \Pi \Gamma}$ and $\mathbf{S}^{J \Pi \Gamma}$ matrices (which are also independent of $M$ ) are determined from a knowledge of the matrix $\mathbf{C}^{J \Pi \Gamma}$ whose elements are the coefficients $C_{\lambda b_{\lambda}}^{J \Pi \Gamma \lambda^{\prime} b_{\lambda^{\prime}}^{\prime}}$ of Eq. (26). To that effect we rewrite Eq. (10) in the form

$$
\hat{H}_{\lambda}=\hat{H}_{\lambda}^{(1,2)}\left(\mathbf{r}_{\lambda}^{(1)}, \mathbf{r}_{\lambda}^{(2)}\right)+\hat{H}_{\lambda}^{\text {int }}\left(r_{\lambda}^{(1)}, r_{\lambda}^{(2)}, \mathbf{r}_{\lambda}^{(3)}, \gamma_{\lambda}\right),
$$

where for the diatom-diatom arrangement channels

$$
\begin{aligned}
& \hat{H}_{\lambda}^{(1,2)} \equiv \hat{H}_{\lambda}^{(1)}\left(\mathbf{r}_{\lambda}^{(1)}\right)+\hat{H}_{\lambda}^{(2)}\left(\mathbf{r}_{\lambda}^{(2)}\right), \\
& \hat{H}_{\lambda}^{(i)} \equiv-\frac{\hbar^{2}}{2 \mu r_{\lambda}^{(i)}} \frac{\partial^{2}}{\left(\partial r_{\lambda}^{(i)}\right)^{2}} r_{\lambda}^{(i)}+\frac{\left[\hat{j}_{\lambda}^{(i)}\left(\hat{\mathbf{r}}_{\lambda}^{(i)}\right)\right]^{2}}{2 \mu\left(r_{\lambda}^{(i)}\right)^{2}}+V_{\lambda}^{(i)}\left(r_{\lambda}^{(i)}\right), \\
& i=1,2 \text {, } \\
& \hat{H}_{\lambda}^{\mathrm{int}} \equiv-\frac{\hbar^{2}}{2 \mu r_{\lambda}^{(3)}} \frac{\partial^{2}}{\left(\partial r_{\lambda}^{(3)}\right)^{2}} r_{\lambda}^{(3)}+\frac{\hat{l}_{\mathrm{sf}}^{2}\left(\hat{\mathbf{r}}_{\lambda}^{(3)}\right)}{2 \mu\left(r_{\lambda}^{(3)}\right)^{2}} \\
& +V_{\lambda}^{\mathrm{int}}\left(r_{\lambda}^{(1)}, r_{\lambda}^{(2)}, r_{\lambda}^{(3)}, \gamma_{\lambda}\right) \\
& V_{\lambda}^{\mathrm{int}} \equiv V\left(r_{\lambda}^{(1)}, r_{\lambda}^{(2)}, r_{\lambda}^{(3)}, \gamma_{\lambda}\right)-V_{\lambda}^{(1)}\left(r_{\lambda}^{(1)}\right)-V_{\lambda}^{(2)}\left(r_{\lambda}^{(2)}\right) .
\end{aligned}
$$

In Eq. (28b) $V_{\lambda}^{(i)}\left(r_{\lambda}^{(i)}\right)(i=1,2)$ are the diatomic potentials, and $V\left(r_{\lambda}^{(1)}, r_{\lambda}^{(2)}, r_{\lambda}^{(3)}, \gamma_{\lambda}\right)$ in Eq. (28d) is the total tetraatomic potential that depends on the six internal coordinates of the system. For the atom-triatom arrangement channels the terms in Eq. (27) are defined as

$$
\begin{aligned}
\hat{H}_{\lambda}^{(1,2) \equiv} \equiv & -\frac{\hbar^{2}}{2 \mu r_{\lambda}^{(1)}} \frac{\partial^{2}}{\left(\partial r_{\lambda}^{(1)}\right)^{2}} r_{\lambda}^{(1)}-\frac{\hbar^{2}}{2 \mu r_{\lambda}^{(2)}} \frac{\partial^{2}}{\left(\partial r_{\lambda}^{(2)}\right)^{2}} r_{\lambda}^{(2)} \\
& +\frac{\left[\hat{j}_{\lambda}^{(1)}\left(\hat{\mathbf{r}}_{\lambda}^{(1)}\right)\right]^{2}}{2 \mu\left(r_{\lambda}^{(1)}\right)^{2}}+\frac{\left[\hat{j}_{\lambda}^{(2)}\left(\hat{\mathbf{r}}_{\lambda}^{(2)}\right)\right]^{2}}{2 \mu\left(r_{\lambda}^{(2)}\right)^{2}} \\
& +V_{\lambda}^{(1,2)}\left(r_{\lambda}^{(1)}, r_{\lambda}^{(2)}, \gamma_{\lambda}^{(1,2)}\right) \\
\hat{H}_{\lambda}^{\mathrm{int}} \equiv & -\frac{\hbar^{2}}{2 \mu r_{\lambda}^{(3)}} \frac{\partial^{2}}{\left(\partial r_{\lambda}^{(3)}\right)^{2}} r_{\lambda}^{(3)}+\frac{\hat{l}_{\mathrm{sf}}^{2}\left(\hat{\mathbf{r}}_{\lambda}^{(3)}\right)}{2 \mu\left(r_{\lambda}^{(3)}\right)^{2}} \\
& +V_{\lambda}^{\mathrm{int}}\left(r_{\lambda}^{(1)}, r_{\lambda}^{(2)}, r_{\lambda}^{(3)}, \gamma_{\lambda}\right), \\
V_{\lambda}^{\mathrm{int} \equiv} \equiv & V\left(r_{\lambda}^{(1)}, r_{\lambda}^{(2)}, r_{\lambda}^{(3)}, \gamma_{\lambda}\right)-V_{\lambda}^{(1,2)}\left(r_{\lambda}^{(1)}, r_{\lambda}^{(2)}, \gamma_{\lambda}^{(1,2)}\right)
\end{aligned}
$$

where $V_{\lambda}^{(1,2)}$ in Eq. (29b) is the triatomic potential which depends on the three internal coordinates $\left(r_{\lambda}^{(1)}, r_{\lambda}^{(2)}, \gamma_{\lambda}^{(1,2)}\right)$. Common to both the diatom-diatom and atom-triatom cases is the fact that the Hamiltonian $\hat{H}_{\lambda}^{\text {int }}$ (which describes the relative motion of the two reactants or the two products) includes an interaction potential $V_{\lambda}^{\text {int }}$ and the orbital angular momentum operator $\hat{l}_{\mathrm{sf}}^{2}\left(\hat{\mathbf{r}}_{\lambda}^{(3)}\right)$ associated with the relative motion of these species.

We now expand the $\Psi_{\lambda^{\prime} b_{\lambda^{\prime}}^{\prime}}^{J M \Pi \Gamma}$ of Eq. (26) in the asymptotic region according to

$$
\Psi_{\lambda^{\prime} b_{\lambda^{\prime}}^{\prime}}^{J M \Pi \Gamma} \sim \sum_{\lambda b_{\lambda}} \frac{U_{\lambda b_{\lambda}}^{J \Pi \Gamma \lambda^{\prime} b_{\lambda^{\prime}}^{\prime}\left(r_{\lambda}^{(3)}\right)}}{r_{\lambda}^{(3)}} \Phi_{\lambda b_{\lambda}}^{J M}\left(\mathbf{r}_{\lambda}^{(1)}, \mathbf{r}_{\lambda}^{(2)}, \hat{\mathbf{r}}_{\lambda}^{(3)}\right),
$$

where $U_{\lambda b_{\lambda}}^{J \Pi \Gamma \lambda^{\prime} b_{\lambda^{\prime}}^{\prime}}$ denotes a set of (still undetermined) radial functions and the molecular basis functions $\Phi_{\lambda b_{\lambda}}^{J M}$ are simultaneous solutions of a set of arrangement-channel-dependent eigenvalue-eigenfunction equations. The $\Pi$ dependence of the $U_{\lambda b_{\lambda}}^{J \Pi \Gamma \lambda^{\prime} b_{\lambda^{\prime}}^{\prime}}$ stems from that in $C_{\lambda b_{\lambda}}^{J \Pi \Gamma \lambda^{\prime} b_{\lambda^{\prime}}^{\prime}}$ of Eq. (26), which itself resulted from the $\Pi$ dependence of the LHSF in the strong interaction region. The latter is imposed in order to achieve decoupling between the $\Pi=0$ and $\Pi=1$ solutions which is extremely important as it decreases the computation time for the partial wave scattering equations by a factor of 4. In the diatom-diatom case these equations are

$$
\begin{aligned}
& \hat{J}^{2} \Phi_{\lambda b_{\lambda}}^{J M}=\hbar^{2} J(J+1) \Phi_{\lambda b_{\lambda}}^{J M}, \\
& \hat{J}_{\mathrm{sf}_{z}} \Phi_{\lambda b_{\lambda}}^{J M}=\hbar M \Phi_{\lambda b_{\lambda}}^{J M}, \\
& \hat{J}_{\mathrm{bf}_{1 z}} \Phi_{\lambda b_{\lambda}}^{J M}=\hat{J}_{\mathrm{bf}_{2 z}} \Phi_{\lambda b_{\lambda}}^{J M}=\hbar \Omega_{\lambda} \Phi_{\lambda b_{\lambda}}^{J M}, \\
& \hat{J}_{\lambda}^{2} \Phi_{\lambda b_{\lambda}}^{J M}=\hbar^{2} J_{\lambda}\left(J_{\lambda}+1\right) \Phi_{\lambda b_{\lambda}}^{J M}, \\
& {\left[\hat{\mathbf{j}}_{\lambda}^{(i)}\right]^{2} \Phi_{\lambda b_{\lambda}}^{J M}=\hbar^{2} j_{\lambda}^{(i)}\left(j_{\lambda}^{(i)}+1\right) \Phi_{\lambda b_{\lambda}}^{J M}, \quad i=1,2,} \\
& \hat{H}_{\lambda}^{(1,2)} \Phi_{\lambda b_{\lambda}}^{J M}=E_{\lambda \epsilon_{\lambda}} \Phi_{\lambda b_{\lambda}}^{J M},
\end{aligned}
$$

where

$$
\hat{\mathbf{J}}_{\lambda} \equiv \hat{\mathbf{j}}_{\lambda}^{(1)}+\hat{\mathbf{j}}_{\lambda}^{(2)}
$$


and we have used the compact quantum number notation

$$
\begin{aligned}
\lambda b_{\lambda} & \equiv\left\{\lambda \epsilon_{\lambda}, J_{\lambda}, \Omega_{\lambda}\right\} \\
& =\left\{\lambda_{1}, \lambda_{2}, v_{\lambda}^{(1)}, v_{\lambda}^{(2)}, j_{\lambda}^{(1)}, j_{\lambda}^{(2)}, J_{\lambda}, \Omega_{\lambda}\right\} .
\end{aligned}
$$

It should be noticed that the $O z_{\lambda}^{\mathrm{bf}_{1}}$ and $O z_{\lambda}^{\mathrm{bf}_{2}}$ axes and the associated components of the total angular momentum vector operator are the same, as indicated in Eq. (31c). In the atomtriatom case the eigenvalue equations are the same except that Eqs. (31d), (31e), and (32) are replaced by the single equation

$$
\left[\hat{\mathbf{j}}_{\lambda}^{(1)}\right]^{2} \Phi_{\lambda b_{\lambda}}^{J M}=\hbar^{2} j_{\lambda}^{(1)}\left(j_{\lambda}^{(1)}+1\right) \Phi_{\lambda b_{\lambda}}^{J M}
$$

with the corresponding compact quantum number indices now being

$$
\lambda b_{\lambda} \equiv\left\{\lambda \epsilon_{\lambda}, \Omega_{\lambda}\right\}=\left\{\lambda_{1}, \lambda_{2}, \mathbf{v}_{\lambda}^{(1)}, j_{\lambda}^{(1)}, \Omega_{\lambda}\right\} .
$$

Equation (31) and its atom-triatom counterpart will clearly be satisfied if we express the dependence of $\Phi_{\lambda b_{\lambda}}^{J M}$ on the Euler angles as

$$
\begin{aligned}
\Phi_{\lambda b_{\lambda}}^{J M}\left(\mathbf{r}_{\lambda}^{(1)}, \mathbf{r}_{\lambda}^{(2)}, \hat{\mathbf{r}}_{\lambda}^{(3)}\right) & \equiv D_{M \Omega_{\lambda}}^{J}\left(\mathbf{a}_{\lambda}^{\mathrm{bf}_{1}}\right) \Phi_{\lambda b_{\lambda}}^{\mathrm{bf}_{1}}\left(\mathbf{r}_{\lambda}^{(1)}, \mathbf{r}_{\lambda}^{(2)}\right) \\
& \equiv D_{M \Omega_{\lambda}}^{J}\left(\mathbf{a}_{\lambda}^{\mathrm{bf}_{2}}\right) \Phi_{\lambda b_{\lambda}}^{\mathrm{bf}_{2}}\left(r_{\lambda}^{(1)}, r_{\lambda}^{(2)}, \boldsymbol{\gamma}_{\lambda}\right),
\end{aligned}
$$

where $\Phi_{\lambda b_{\lambda}}^{\mathrm{bf}_{i}}$ are the body-fixed molecular wave functions, to be specified later. To explicitly define the radial expansion

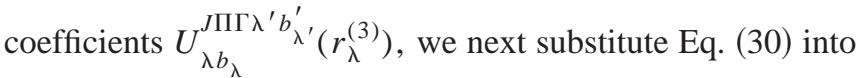
Eq. (25a), multiply the result by $\Phi_{\lambda b_{\lambda}}^{J M^{*}}$ and finally integrate over all coordinates except $r_{\lambda}^{(3)}$. Using the definitions in Eq. (27), Eq. (31), and Eq. (36) this can be shown to give the following coupled system of ordinary differential equations in $U_{\lambda b_{\lambda}}^{J \Pi \Gamma \lambda^{\prime} b_{\lambda^{\prime}}^{\prime}}\left(r_{\lambda}^{(3)}\right)$

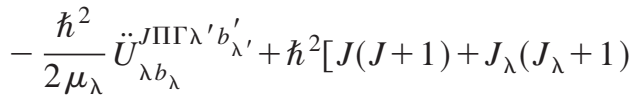

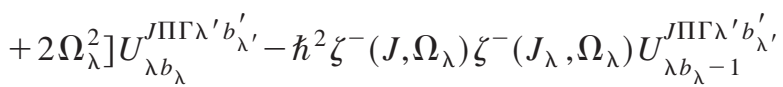

$$
\begin{aligned}
& -\hbar^{2} \zeta^{+}\left(J, \Omega_{\lambda}\right) \zeta^{+}\left(J_{\lambda}, \Omega_{\lambda}\right) U_{\lambda b_{\lambda}+1}^{J \Pi \Gamma \lambda^{\prime} b_{\lambda^{\prime}}^{\prime}} \\
& +\sum_{\lambda^{\prime \prime} b_{\lambda^{\prime \prime}}^{\prime \prime}}\left\langle\Phi_{\lambda b_{\lambda}}^{J M}\left|V_{\lambda}^{\mathrm{int}}\right| \Phi_{\lambda^{\prime \prime} b_{\lambda^{\prime \prime}}^{\prime \prime}}^{J M}\right\rangle U_{\lambda^{\prime \prime} b_{\lambda^{\prime \prime}}^{\prime \prime}}^{J \Pi \Gamma \lambda^{\prime} b_{\lambda^{\prime}}^{\prime}} \\
& \sim\left(E-E_{\lambda \epsilon_{\lambda}}\right) U_{\lambda b_{\lambda}}^{J \Pi \Gamma \lambda^{\prime} b_{\lambda^{\prime}}^{\prime}}
\end{aligned}
$$

where $\ddot{U}_{\lambda b_{\lambda}}^{J \Pi \Gamma \lambda, b_{\lambda^{\prime}}^{\prime}}$ denotes the second order derivative with respect to $r_{\lambda}^{(3)}, \lambda b_{\lambda} \pm 1 \equiv\left\{\lambda \epsilon_{\lambda}, J_{\lambda}, \Omega_{\lambda} \pm 1\right\}$ and $\zeta^{ \pm}\left(J, \Omega_{\lambda}\right)$ $\equiv \sqrt{J(J+1)-\Omega_{\lambda}\left(\Omega_{\lambda} \pm 1\right)}$. Furthermore, since the interaction potential, $V_{\lambda}^{\text {int }}\left(r_{\lambda}^{(1)}, r_{\lambda}^{(2)}, r_{\lambda}^{(3)}, \gamma_{\lambda}\right)$, is invariant under space rotations (i.e., is independent of $\mathbf{a}_{\lambda}^{\mathrm{bf}_{2}}$ ) the potential coupling terms in Eq. (37) simplify to
$\left\langle\Phi_{\lambda b_{\lambda}}^{J M}\left|V_{\lambda}^{\mathrm{int}}\right| \Phi_{\lambda^{\prime} b_{\lambda^{\prime}}^{\prime}}^{J M}\right\rangle=\left\langle D_{M \Omega_{\lambda}}^{J} \mid D_{M \Omega_{\lambda^{\prime}}^{\prime}}^{J}\right\rangle\left\langle\Phi_{\lambda b_{\lambda}}^{\mathrm{bf}_{2}}\left|V_{\lambda}^{\mathrm{int}}\right| \Phi_{\lambda^{\prime} b_{\lambda^{\prime}}^{\prime}}^{\mathrm{bf}_{2}}\right\rangle$,

$$
=\delta_{\lambda, \lambda^{\prime}}\left\langle D_{M \Omega_{\lambda}}^{J} \mid D_{M \Omega_{\lambda}^{\prime}}^{J}\right\rangle\left\langle\Phi_{\lambda b_{\lambda}}^{\mathrm{bf}_{2}}\left|V_{\lambda}^{\mathrm{int}}\right| \Phi_{\lambda b_{\lambda^{\prime}}^{\prime}}^{\mathrm{bf}_{2}}\right\rangle,
$$

$$
=\frac{8 \pi^{2}}{2 J+1} \delta_{\Omega_{\lambda}, \Omega_{\lambda}^{\prime}}\left\langle\Phi_{\lambda b_{\lambda}}^{\mathrm{bf}_{2}}\left|V_{\lambda}^{\mathrm{int}}\right| \Phi_{\lambda b_{\lambda}^{\prime}}^{\mathrm{bf}_{2}}\right\rangle,
$$

where Eq. (38b) follows from the fact that the body-fixed molecular wave functions are localized in the arrangement channels and Eq. (38c) is a simple consequence of the orthogonality of the Wigner rotation matrices. Thus we conclude that in the body-fixed total angular momentum representation of Eq. (30) the potential energy matrix is diagonal in $\Omega_{\lambda}$ and independent of both $J$ and $M$, whereas the kinetic energy part is tri-diagonal in $\Omega_{\lambda}$ and diagonal in the remaining quantum numbers $\left(\left\{v_{\lambda}^{(1)}, v_{\lambda}^{(2)}, j_{\lambda}^{(1)}, j_{\lambda}^{(2)}, J_{\lambda}\right\}\right.$ or $\left\{\mathbf{v}_{\lambda}^{(1)}, j_{\lambda}^{(1)}\right\}$ depending on the arrangement channel considered).

The next important step in determining $\mathbf{R}^{J \Pi \Gamma}$ is to consider the asymptotic limit of Eq. (37) for which the interaction potential can be assumed to have vanished and then express these body-fixed $U_{\lambda b_{\lambda}}^{J \Pi \Gamma \lambda^{\prime} b_{\lambda^{\prime}}^{\prime}}\left(r_{\lambda}^{(3)}\right)$ in terms of linear combinations of the known analytical solutions to the corresponding space-fixed equation (also in the absence of the potential coupling term). However, it will not be assumed that the Coriolis coupling terms represented by $U_{\lambda b_{\lambda} \pm 1}^{J \Pi \Gamma \lambda^{\prime} b_{\lambda^{\prime}}^{\prime}}$ in Eq. (37) have become negligible. These analytical solutions of the asymptotic form of Eq. (37) are obtained in the Appendix. Since one can express them as real standing waves or as complex propagating spherical waves, two different approaches can be adopted at this point. Throughout the remaining of this paper we shall outline both in parallel since they are related in a simple manner, and finally choose one for convenience in the numerical implementations. In the asymptotic limit of Eq. (37) we expand the linearly independent solutions as

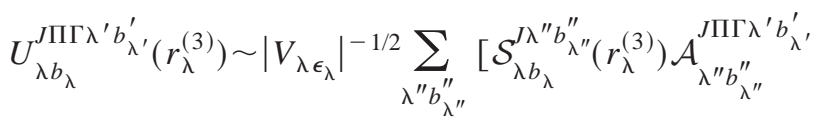

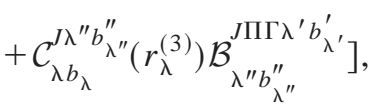

$$
\begin{aligned}
& U_{\lambda b_{\lambda}}^{J \Pi \Gamma \lambda^{\prime} b_{\lambda^{\prime}}^{\prime}}\left(r_{\lambda}^{(3)}\right) \sim\left|V_{\lambda \epsilon_{\lambda}}\right|^{-1 / 2} \sum_{\lambda^{\prime \prime} b_{\lambda^{\prime \prime}}^{\prime \prime}}\left[\mathcal{I}_{\lambda b_{\lambda}}^{J \lambda^{\prime \prime} b_{\lambda^{\prime \prime}}^{\prime \prime}}\left(r_{\lambda}^{(3)}\right) \mathcal{C}_{\lambda^{\prime \prime} b_{\lambda^{\prime \prime}}^{\prime \prime}}^{J \Pi \Gamma \lambda^{\prime} b_{\lambda^{\prime}}^{\prime}}\right.
\end{aligned}
$$

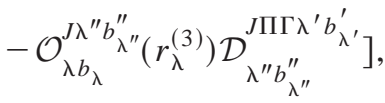

where $V_{\lambda \epsilon_{\lambda}}$ are the channel velocities, $\mathcal{A}^{J \Pi \Gamma}, \mathcal{B}^{J \Pi \Gamma}, \mathcal{C}^{J \Pi \Gamma}$, $\mathcal{D}^{J \Pi \Gamma}$ are the constant ( $\rho$-independent) expansion coefficient 
matrices for the analytical functions $\mathcal{S}_{\lambda b_{\lambda}}^{J \lambda^{\prime \prime} b^{\prime \prime}}\left(r_{\lambda}^{(3)}\right)$, $\mathcal{C}_{\lambda b_{\lambda}}^{J \lambda^{\prime \prime} b^{\prime \prime}}\left(r_{\lambda}^{(3)}\right), \mathcal{I}_{\lambda b_{\lambda}}^{J \lambda^{\prime \prime} b_{\lambda^{\prime \prime}}}\left(r_{\lambda}^{(3)}\right)$ and $\mathcal{O}_{\lambda b_{\lambda}}^{J \lambda^{\prime \prime} b_{\lambda^{\prime \prime}}}\left(r_{\lambda}^{(3)}\right)$ derived in the Appendix, Eq. (A15).

The pairs of square matrices, $\left\{\mathcal{A}^{J \Pi \Gamma}, \mathcal{B}^{J \Pi \Gamma}\right\}$ and $\left\{\mathcal{C}^{J \Pi \Gamma}, \mathcal{D}^{J \Pi \Gamma}\right\}$, introduced in Eq. (39), depend on the choice of initial conditions adopted in the solution of the differential equation satisfied by the matrix $\mathbf{C}^{J \Pi \Gamma \lambda}$ defined by the $\mathcal{C}_{\lambda^{\prime} b_{\lambda^{\prime}}^{\prime}}^{J \Pi \Gamma \lambda b_{\lambda}}$ coefficients in Eq. (26). The $\Phi_{\lambda b_{\lambda}}^{J M}$ in the 1.h.s. of that equation are obtained by integrating the Schrödinger equation starting with small values of $\rho$ as indicated prior to Eq. (26). Other than requiring that $\Phi_{\lambda b_{\lambda}}^{J M}$ approach zero as $\rho \sim 0$, arbitrary initial conditions regarding its $\rho$ derivatives are used. However, as a result of the linearity of that Schrödinger equation the "ratios" between those two pairs of matrices, namely,

$$
\begin{aligned}
& \mathbf{R}^{J \Pi \Gamma}=\mathcal{B}^{J \Pi \Gamma} \cdot\left[\mathcal{A}^{J \Pi \Gamma}\right]^{-1}, \\
& \mathbf{S}^{J \Pi \Gamma}=\mathcal{D}^{J \Pi \Gamma} \cdot\left[\mathcal{C}^{J \Pi \Gamma}\right]^{-1},
\end{aligned}
$$

are unique, i.e., are independent of those $\Phi_{\lambda b_{\lambda}}^{J M}$ initial conditions. Throughout the remaining of this paper we shall refer to the standing wave basis set of Eqs. (A10) and (A11) [appearing in Eq. (39a)] as the R-matrix representation and the spherical waves of Eqs. (A12) and (A13) [appearing in Eq. (39b)] as the $\mathbf{S}$-matrix representation.

Whereas the S-matrix representation with spherical incoming and outgoing waves is far more intuitive physically than the formally equivalent $\mathbf{R}$-matrix standing wave representation, the latter does offer a more attractive formulation due to the numerical convenience that $\mathbf{R}$ is real. The stateto-state cross sections depend only on the open-row, opencolumn part of the $\mathbf{S}$-matrix, denoted by $\mathbf{S}_{o o}^{J \Pi \Gamma}$. Its relation to the corresponding open-open part $\mathbf{R}_{o o}^{J \Pi \Gamma}$ of the $\mathbf{R}$-matrix is given by ${ }^{41}$

$$
\mathbf{S}_{o o}^{J \Pi \Gamma}=\left[\mathbf{I}+i \mathbf{R}_{o o}^{J \Pi \Gamma}\right]^{-1} \cdot\left[\mathbf{I}-i \mathbf{R}_{o o}^{J \Pi \Gamma}\right] .
$$

In the rest of this paper we obtain expressions for the $\mathbf{R}$ - and $\mathbf{S}$-matrices in terms of any complete set of linearly independent solutions to the time-independent Schrödinger equation.

\section{B. Calculation of the reactance and scattering matrices}

We now derive explicit expressions for the reactance $(\mathbf{R})$ and scattering $(\mathbf{S})$ matrices in terms of the expansion coefficients $C_{\lambda b_{\lambda}}^{J \Pi \Gamma \lambda^{\prime} b_{\lambda^{\prime}}^{\prime}}$ defined by Eq. (26). The strategy is simply to equate $\Psi_{\lambda^{\prime} b_{\lambda^{\prime}}^{\prime}}^{J M \Gamma}$ given by that equation at a fixed large value of the hyperradius, $\bar{\rho}$, with its value given by Eq. (30) with $U_{\lambda b_{\lambda}}^{J \Pi \Gamma \lambda^{\prime} b_{\lambda^{\prime}}^{\prime}}$ expressed by Eq. (39), followed by an integration over all angles. It should be noted that it is equally possible to make this projection at a fixed value of $r_{\lambda}^{(3)}$ (as opposed to $\rho$ ), but this strategy is complicated by the fact that the corresponding wave function cuts across several values of $\bar{\rho}$, i.e., the fixed- $r_{\lambda}^{(3)}$ values of $\Psi_{\lambda^{\prime} b_{\lambda^{\prime}}^{\prime}}^{J M \Pi \Gamma}$ have contributions from
$\Phi_{J \Pi \Gamma \lambda b_{\lambda}}^{\mathrm{LHSF}}$ defined in different spherical sections. Both derivations have been worked out, but we shall restrict ourself to the compact fixed- $\bar{\rho}$ version. Before this can be done, however, it is necessary to switch from the principal moment of inertia Cartesian axes used in Eq. (26) to the Jacobi bodyfixed-2 axes used in Eq. (30). This is accomplished by means of the transformation relations derived in Sec. II D. From Eqs. (22), (26), (30), and (36) we obtain the following equalities:

$$
\begin{aligned}
& \Psi_{\lambda^{\prime} b_{\lambda^{\prime}}^{\prime}}^{J M \Pi \Gamma}=\rho^{-4} \sum_{\lambda b_{\lambda}} C_{\lambda b_{\lambda}}^{J \Pi \Gamma \lambda^{\prime} b_{\lambda^{\prime}}^{\prime}}(\rho ; \bar{\rho}) \\
& \times D_{M \Omega_{\lambda}}^{J}\left(\mathbf{a}_{\lambda}^{\tau}\right) \Phi_{J \Pi \Gamma \lambda b_{\lambda}}^{\mathrm{LHSF}}\left(\boldsymbol{\eta}_{\lambda}, \gamma_{\lambda} ; \overline{\boldsymbol{\rho}}\right),
\end{aligned}
$$

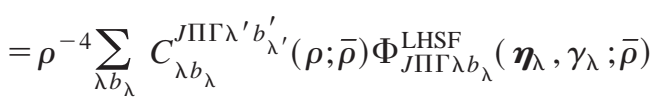

$$
\begin{aligned}
& \times \sum_{K_{\lambda}} D_{M K_{\lambda}}^{J}\left(\mathbf{a}_{\lambda}^{\mathrm{bf}_{2}}\right) D_{\Omega_{\lambda} K_{\lambda}}^{J}\left(\boldsymbol{\alpha}_{\lambda}\left(\boldsymbol{\eta}_{\lambda}, \gamma_{\lambda}\right)\right), \\
& =\sum_{\lambda b_{\lambda}} \frac{U_{\lambda b_{\lambda}}^{J \Pi \Gamma \lambda^{\prime} b_{\lambda^{\prime}}^{\prime}\left(r_{\lambda}^{(3)}\right)}}{r_{\lambda}^{(3)}} D_{M \Omega_{\lambda}}^{J}\left(\mathbf{a}_{\lambda}^{\mathrm{bf}_{2}}\right) \Phi_{\lambda b_{\lambda}}^{\mathrm{bf}_{2}} \\
& \times\left(r_{\lambda}^{(1)}, r_{\lambda}^{(2)}, \gamma_{\lambda}\right) .
\end{aligned}
$$

We next multiply Eqs. (42b) and (42c) by $\Phi_{J \Pi \Gamma \lambda b_{\lambda}^{-}}^{\mathrm{LHSF^{* }}}\left(\boldsymbol{\eta}_{\lambda}^{-}, \boldsymbol{\gamma}_{\lambda}^{-} ; \overline{\boldsymbol{\rho}}\right) D_{M \Omega_{\lambda}^{-}}^{J^{*}}\left(\mathbf{a}_{\lambda}^{\mathrm{bf}_{2}}\right)$, use Eq. (11) to expand $\left(r_{\lambda}^{(1)}, r_{\lambda}^{(2)}, r_{\lambda}^{(3)}\right)$ in terms of $\left(\rho, \boldsymbol{\eta}_{\lambda}\right)$ and finally integrate the resulting expression over all eight hyperangles $\boldsymbol{\eta}_{\lambda}, \boldsymbol{\gamma}_{\lambda}$, and $\mathbf{a}_{\lambda}^{\mathrm{bf}_{2}}$ with $\rho$ set equal to $\bar{\rho}$. After the substitution of Eq. (39) into Eq. (42c) and performing some straightforward but lengthy algebraic manipulations we arrive at the matrix equations

$$
\begin{aligned}
& \mathbf{O}^{J} \mathbf{C}^{J \Pi \Gamma}=\mathcal{S}^{J} \mathcal{A}^{J \Pi \Gamma}-\mathcal{C}^{J} \mathcal{B}^{J \Pi \Gamma}, \\
& \mathbf{O}^{J} \mathbf{C}^{J \Pi \Gamma}=\mathcal{I}^{J} \mathcal{C}^{J \Pi \Gamma}-\mathcal{O}^{J} \mathcal{D}^{J \Pi \Gamma} .
\end{aligned}
$$

The overlap matrices $\mathbf{O}^{J}$ and $\mathcal{X}^{J} \equiv\left\{\mathcal{S}^{J}, \mathcal{C}^{J}, \mathcal{I}^{J}, \mathcal{O}^{J}\right\}$ which appear in these expressions are defined by

$$
\begin{aligned}
& {\left[O^{J}\right]_{\overline{\lambda b_{\lambda}^{-}}}^{\lambda b_{\lambda}}=\delta_{\bar{\lambda}}^{\lambda} \int d \boldsymbol{\eta}_{\bar{\lambda}} \int d \gamma_{\bar{\lambda}} \Phi_{J \lambda b_{\bar{\lambda}}^{-}}^{\mathrm{LHSF}^{*}}\left(\boldsymbol{\eta}_{\bar{\lambda}}, \gamma_{\bar{\lambda}} ; \bar{\rho}\right)} \\
& \times \Phi_{J \lambda b_{\bar{\lambda}}^{-}}^{\mathrm{LHSF}}\left(\boldsymbol{\eta}_{\bar{\lambda}}, \gamma_{\bar{\lambda}} ; \bar{\rho}\right) D_{\Omega_{\bar{\lambda}}^{J_{\bar{\lambda}}} \bar{\Omega}_{\bar{\lambda}}^{*}}\left(\boldsymbol{\alpha}_{\bar{\lambda}}^{-}\left(\boldsymbol{\eta}_{\bar{\lambda}}, \gamma_{\bar{\lambda}}^{-}\right)\right),
\end{aligned}
$$

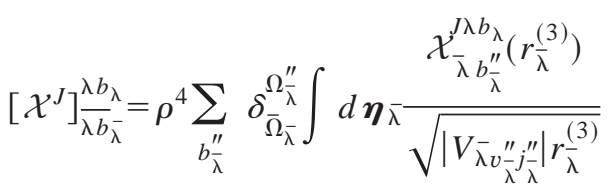

$$
\begin{aligned}
& \times \int d \gamma_{\bar{\lambda}}^{-} \Phi_{J \lambda b_{\bar{\lambda}}}^{\mathrm{LHSF}}\left(\boldsymbol{\eta}_{\bar{\lambda}}, \gamma_{\bar{\lambda}} ; \bar{\rho}\right) \Phi_{\bar{\lambda} b_{\bar{\lambda}}^{\prime \prime}}^{\mathrm{bf}_{2}}\left(r_{\bar{\lambda}}^{(1)}, r_{\bar{\lambda}}^{(2)}, \gamma_{\bar{\lambda}}\right),
\end{aligned}
$$

in which $\left(r_{\lambda}^{(1)}, r_{\lambda}^{(2)}, r_{\lambda}^{(3)}\right)$ are the functions of $\left(\rho, \boldsymbol{\eta}_{\lambda}\right)$ defined by Eq. (11), and the integrals are over the five internal Delves hyperangles $\left(\boldsymbol{\eta}_{\lambda}, \boldsymbol{\gamma}_{\lambda}\right)$. Note also that the symbol $\mathcal{X}$ in Eq. (44b) is identical to the one in Eq. (A15) and can assume the values $\mathcal{S}, \mathcal{C}, \mathcal{I}$, or $\mathcal{O}$ corresponding to the two sets of 
asymptotic radial functions in respectively the $R$ - or $S$-matrix representation. In addition, since $\bar{\rho}$ is sufficiently large for the potential coupling matrix elements between different arrangement channel regions to have vanished, the corresponding $\Phi_{J \lambda b_{\lambda}^{-}}^{\mathrm{LHSF}^{*}}$ are independent of both $\Pi$ and $\Gamma$ as they are completely localized in those regions. As a result, the $\mathbf{O}^{J}$ and $\boldsymbol{\mathcal { X }}^{J}$ are also independent of $\Pi$ and $\Gamma$.

Left-multiplying Eq. (43) by $\left[\mathbf{O}^{J}\right]^{-1}$ and taking the derivative of the resulting expression with respect to $\rho$ we now get

$$
\begin{aligned}
\frac{d}{d \rho}\left[\mathbf{C}^{J \Pi \Gamma}\right] \equiv \dot{\mathbf{C}}^{J \Pi \Gamma} & =\left[\mathbf{O}^{J}\right]^{-1}\left(\dot{\mathcal{S}}^{J}-\dot{\mathcal{C}}^{J} \mathbf{R}^{J \Pi \Gamma}\right) \mathcal{A}^{J \Pi \Gamma}, \\
& =\left[\mathbf{O}^{J}\right]^{-1}\left(\dot{\mathcal{I}}^{J}-\dot{\mathcal{O}}^{J} \mathbf{S}^{J \Pi \Gamma}\right) \mathcal{C}^{J \Pi \Gamma},
\end{aligned}
$$

where the dot above the matrices denote $\rho$-derivatives. We now right-multiply this expression by $\left[\mathbf{C}^{J \Pi \Gamma}\right]^{-1}$ to get

$$
\begin{aligned}
\mathbf{Y}^{J \Pi \Gamma} & \equiv \dot{\mathbf{C}}^{J \Pi \Gamma}\left[\mathbf{C}^{J \Pi \Gamma}\right]^{-1} \\
& =\left[\mathbf{O}^{J}\right]^{-1}\left(\dot{\mathcal{S}}^{J}-\dot{\mathcal{C}}^{J} \mathbf{R}^{J \Pi \Gamma}\right)\left(\mathcal{S}^{J}-\mathcal{C}^{J} \mathbf{R}^{J \Pi \Gamma}\right)^{-1} \mathbf{O}^{J}, \\
& =\left[\mathbf{O}^{J}\right]^{-1}\left(\dot{\mathcal{I}}^{J}-\dot{\mathcal{O}}^{J} \mathbf{S}^{J \Pi \Gamma}\right)\left(\mathcal{I}^{J}-\mathcal{O}^{J} \mathbf{S}^{J \Pi \Gamma}\right)^{-1} \mathbf{O}^{J},
\end{aligned}
$$

where $\mathbf{Y}^{J \Pi \Gamma}$ is by definition the logarithmic derivative of $\mathbf{C}^{J \Pi \Gamma}$. This leads to the following expressions for the reactance and scattering matrices:

$$
\begin{aligned}
& \mathbf{R}^{J \Pi \Gamma}=\left(\mathcal{C}^{J}-\overline{\mathbf{Y}}^{J \Pi \Gamma} \dot{\mathcal{C}}^{J}\right)^{-1}\left(\mathcal{S}^{J}-\overline{\mathbf{Y}}^{J \Pi \Gamma} \dot{\mathcal{S}}^{J}\right), \\
& \mathbf{S}^{J \Pi \Gamma}=\left(\mathcal{O}^{J}-\overline{\mathbf{Y}}^{J \Pi \Gamma} \dot{\mathcal{O}}^{J}\right)^{-1}\left(\mathcal{I}^{J}-\overline{\mathbf{Y}}^{J \Pi \Gamma} \dot{\mathcal{I}}^{J}\right),
\end{aligned}
$$

where we have defined the similarity-transformed logarithmic derivative matrix, $\overline{\mathbf{Y}}^{J \Pi \Gamma}$, by

$$
\overline{\mathbf{Y}}^{J \Pi \Gamma} \equiv \mathbf{O}^{J} \mathbf{Y}^{J \Pi \Gamma}\left[\mathbf{O}^{J}\right]^{-1} .
$$

As mentioned in Sec. III A, the R-matrix real standing wave representation offers a more attractive formulation than the formally equivalent $\mathbf{S}$-matrix complex propagating spherical wave representation due to the numerical convenience of being calculable using real (as opposed to complex) arithmetic. However, to compute state-to-state differential cross-sections the open-open submatrix, $\mathbf{S}_{o o}^{J \Pi \Gamma}$, is needed, and can be obtained from $\mathbf{R}_{o o}^{J \Pi \Gamma}$ by means of Eq. (41). To optimize this approach we partition Eq. (47a) into open-open, openclosed, closed-open, and closed-closed submatrices according to the closed and open nature of the local hyperspherical surface functions $\Phi_{J \Pi \Gamma \lambda b_{\lambda}}^{\mathrm{LHSF}}$ which appear in Eq. (44), according to

$$
\begin{aligned}
\left(\begin{array}{ll}
\mathbf{R}_{o o}^{J \Pi \Gamma} & \mathbf{R}_{o c}^{J \Pi \Gamma} \\
\mathbf{R}_{c o}^{J \Pi \Gamma} & \mathbf{R}_{c c}^{J \Pi \Gamma}
\end{array}\right)= & \left(\begin{array}{cc}
\mathcal{C}_{o o}^{J}-\overline{\mathbf{Y}}_{o o}^{J \Pi \Gamma} \dot{\mathcal{C}}_{o o}^{J} & -\overline{\mathbf{Y}}_{o c}^{J \Pi \Gamma} \dot{\mathcal{C}}_{c c}^{J} \\
-\overline{\mathbf{Y}}_{c o}^{J \Pi \Gamma} \dot{\mathcal{C}}_{o o}^{J} & \mathcal{C}_{c c}^{J}-\overline{\mathbf{Y}}_{c c}^{J \Pi \Gamma} \dot{\mathcal{C}}_{c c}^{J}
\end{array}\right)^{-1} \\
& \times\left(\begin{array}{cc}
\mathcal{S}_{o o}^{J}-\overline{\mathbf{Y}}_{o o}^{J \Pi \Gamma} \dot{\mathcal{S}}_{o o}^{J} & -\overline{\mathbf{Y}}_{o c}^{J \Pi \Gamma} \dot{\mathcal{S}}_{c c}^{J} \\
-\overline{\mathbf{Y}}_{c o}^{J \Pi \Gamma} \dot{\mathcal{S}}_{o o}^{J} & \mathcal{S}_{c c}^{J}-\overline{\mathbf{Y}}_{c c}^{J \Pi \Gamma} \dot{\mathcal{S}}_{c c}^{J}
\end{array}\right),
\end{aligned}
$$

where we have used the fact that the matrices $\mathcal{X}^{J}$ $\equiv \mathcal{S}^{J}, \mathcal{C}^{J}, \mathcal{I}^{J}, \mathcal{O}^{J}$ are block-diagonal in the open/closed labeling which follows form the definitions in the Appendix [see Eqs. (A10)-(A15)]. Since, by virtue of Eq. (41), the $\mathbf{S}_{o o}^{J \Pi \Gamma}$ submatrix depends only on $\mathbf{R}_{o o}^{J \Pi \Gamma}$, it is clear that the calculation can be simplified somewhat by only computing the left hand block column of $\mathbf{R}^{J \Pi \Gamma}$ in the 1.h.s. of Eq. (49). This leads to the following matrix equation for the open-column submatrix of the $R$-matrix:

$$
\begin{aligned}
\left(\begin{array}{l}
\mathbf{R}_{o o}^{J \Pi \Gamma} \\
\mathbf{R}_{c o}^{J \Pi \Gamma}
\end{array}\right)= & \left(\begin{array}{cc}
\mathcal{C}_{o o}^{J}-\overline{\mathbf{Y}}_{o o}^{J \Pi \Gamma} \dot{\mathcal{C}}_{o o}^{J} & -\overline{\mathbf{Y}}_{o c}^{J \Pi \Gamma} \dot{\mathcal{C}}_{c c}^{J} \\
-\overline{\mathbf{Y}}_{c o}^{J \Pi \Gamma} \dot{\mathcal{C}}_{o o}^{J} & \mathcal{\mathcal { C }}_{c c}^{J}-\overline{\mathbf{Y}}_{c c}^{J \Pi \Gamma} \dot{\mathcal{C}}_{c o}^{J}
\end{array}\right)^{-1} \\
& \times\left(\begin{array}{c}
\mathcal{\mathcal { S }}_{o o}^{J}-\overline{\mathbf{Y}}_{o o}^{J \Pi \Gamma} \dot{\mathcal{S}}_{o o}^{J} \\
-\overline{\mathbf{Y}}_{c o}^{J \Pi \Gamma} \dot{\mathcal{S}}_{o o}^{J}
\end{array}\right)
\end{aligned}
$$

The inverse matrix in Eq. (50) can next be expressed in terms of its submatrices which after some algebraic manipulations leads to the following equation for the open-open submatrix of the reactance matrix:

$$
\begin{aligned}
\mathbf{R}_{o o}^{J \Pi \Gamma}= & {\left[\mathbf{M}_{o o}^{J \Pi \Gamma}\right]^{-1}\left(\mathbf{S}_{o o}^{J}-\overline{\mathbf{Y}}_{o o}^{J \Pi \Gamma} \dot{\mathcal{S}}_{o o}^{J}\right)-\left[\mathbf{M}_{o o}^{J \Pi \Gamma}\right]^{-1} } \\
& \times \overline{\mathbf{Y}}_{o c}^{J \Pi \Gamma} \dot{\mathcal{C}}_{c c}^{J}\left[\mathcal{C}_{c c}^{J}-\overline{\mathbf{Y}}_{c c}^{J \Pi \Gamma} \dot{\mathcal{C}}_{c o}^{J}\right]^{-1} \overline{\mathbf{Y}}_{c o}^{J \Pi \Gamma} \dot{\mathcal{S}}_{o o}^{J},
\end{aligned}
$$

where

$$
\begin{aligned}
\mathbf{M}_{o o}^{J \Pi \Gamma} \equiv & \mathcal{C}_{o o}^{J}-\overline{\mathbf{Y}}_{o o}^{J \Pi \Gamma} \dot{\mathcal{C}}_{o o}^{J} \\
& -\overline{\mathbf{Y}}_{o c}^{J \Pi \Gamma} \dot{\mathcal{C}}_{c c}^{J}\left[\mathcal{C}_{c c}^{J}-\overline{\mathbf{Y}}_{c c}^{J \Pi \Gamma} \dot{\mathcal{C}}_{c c}^{J}\right]^{-1} \overline{\mathbf{Y}}_{c o}^{J \Pi \Gamma} \dot{\mathcal{C}}_{c c}^{J} .
\end{aligned}
$$

In these expressions $\mathbf{Y}^{J \Pi \Gamma}$ is given by Eq. (46), where $\mathbf{C}^{J \Pi \Gamma}$ is obtained from the propagation of the Schrödinger equation which they satisfy, from the inner to the asymptotic region, and $\mathbf{O}^{J}$, defined in Eq. (44a), can be computed numerically from a knowledge of the LHSF and of the Euler angles $\boldsymbol{\alpha}_{\lambda}\left(\boldsymbol{\eta}_{\lambda}, \boldsymbol{\gamma}_{\lambda}\right)$ obtained in Sec. II D. It should be noticed that $\mathbf{C}^{J \Pi \Gamma}$ and $\dot{\mathbf{C}}^{J \Pi \Gamma}$ are not needed separately; it suffices to obtain their logarithmic derivative $Y^{J \Pi \Gamma}$. As a result, the $\rho$-dependent coefficient matrices obtained from the LHSF expansion in the strong and weak interaction regions can be propagated by logarithmic derivative methods.

To evaluate the five-dimensional integrals in Eq. (44b) which define $\mathbf{X}^{J}$, we first have to give explicit expressions for the asymptotic molecular wave functions $\Phi_{\lambda b_{\lambda}}^{\mathrm{bf}_{2}}$. For a tetraatomic system these functions describe either an atom and a triatom or two diatoms. For the atom-triatom case this function assumes the general form

$$
\begin{aligned}
\Phi_{\lambda b_{\lambda}}^{\mathrm{bf}_{2}}\left(r_{\lambda}^{(1)}, r_{\lambda}^{(2)}, \gamma_{\lambda}\right) \\
\quad=\sum_{K_{\lambda}^{(1)}} D_{\Omega_{\lambda} K_{\lambda}^{(1)}}^{J_{\lambda}}\left(0, \gamma_{\lambda}^{(2,3)}, \phi_{\lambda}\right) \Phi_{\lambda_{1} \epsilon_{\lambda}^{(1)} K_{\lambda}^{(1)}}^{\text {triatom }}\left(r_{\lambda}^{(1)}, r_{\lambda}^{(2)}, \gamma_{\lambda}^{(1,2)}\right),
\end{aligned}
$$

where $\Phi_{\lambda_{1} \epsilon_{\lambda}^{(1)} K_{\lambda}^{(1)}}^{\text {triatom }}$ is the internal triatomic wave function for the quantum numbers $\left\{\epsilon_{\lambda}^{(1)}, K_{\lambda}^{(1)}\right\} \equiv\left\{\mathbf{v}_{\lambda}^{(1)}, j_{\lambda}^{(1)}, K_{\lambda}^{(1)}\right\}$, with $\mathbf{v}_{\lambda}^{(1)}$ denoting the quantum numbers associated with the three vibrational degrees of freedom. Here $j_{\lambda}^{(1)}\left(j_{\lambda}^{(1)}+1\right) \hbar^{2}$ and $K_{\lambda}^{(1)} \hbar$ are the eigenvalues of the square of the triatom's total angular momentum operator $\hat{\mathbf{j}}_{\lambda}^{(1)}$ and of its body-fixed component $\hat{j}_{\lambda}^{(1)}$. In Eq. (53) the body-fixed frame for the triatom has been chosen such that $\mathbf{r}_{\lambda}^{(2)}$ points along the $z$-axis and $\mathbf{r}_{\lambda}^{(1)}$ 
lies in the positive $x z$ half-plane. As a result, the transformation from the tetraatomic body-fixed-2 frame, defined in Sec. II A, to this body-fixed triatomic frame is accomplished through a rotation defined by the Euler angles $\left(0, \gamma_{\lambda}^{(2,3)}, \phi_{\lambda}\right)$, where the hyperangle $\phi_{\lambda}$ can be shown to be related to the angles $\gamma_{\lambda}^{(1,3)}, \gamma_{\lambda}^{(2,3)}$ and $\xi_{\lambda}$, defined in Table I, by

$$
\tan \phi_{\lambda} \equiv \frac{\sin \gamma_{\lambda}^{(1,3)} \sin \xi_{\lambda}}{\sin \gamma_{\lambda}^{(1,3)} \cos \gamma_{\lambda}^{(2,3)} \cos \xi_{\lambda}-\cos \gamma_{\lambda}^{(1,3)} \sin \gamma_{\lambda}^{(2,3)}}
$$

In the diatom-diatom case $\Phi_{\lambda b_{\lambda}}^{\mathrm{bf}_{2}}$ is given by

$\Phi_{\lambda b_{\lambda}}^{\mathrm{bf} b_{2}}\left(r_{\lambda}^{(1)}, r_{\lambda}^{(2)}, \gamma_{\lambda}\right)=\Phi_{\lambda_{1} \epsilon_{\lambda}^{(1)}}^{\text {diatom }}\left(r_{\lambda}^{(1)}\right) \Phi_{\lambda_{2} \epsilon_{\lambda}^{(2)}}^{\text {diatom }}\left(r_{\lambda}^{(2)}\right) \mathcal{Y}_{j_{\lambda}^{(1)} j_{\lambda}^{(2)}}^{J_{\lambda} \Omega_{\lambda}}\left(\gamma_{\lambda}\right)$,

where $\Phi_{\lambda_{i} \epsilon_{\lambda}^{(i)}}^{\text {diatom }}$ denotes the internal wave function of diatom $\lambda_{i}$ with quantum numbers $\left\{v_{\lambda}^{(i)} j_{\lambda}^{(i)}\right\} . \mathcal{Y}_{j_{\beta_{1}} j_{\beta_{2}}}^{J_{\beta} \Omega_{\beta}}$ are ArthursDalgarno spherical harmonics which are defined by the Clebsch-Gordan expansion

$$
\begin{aligned}
\mathcal{Y}_{j_{\lambda}^{(1)} j_{\lambda}^{(2)}}^{J_{\lambda} \Omega_{\lambda}}\left(\gamma_{\lambda}\right) \equiv & \sum_{m_{\lambda}^{(1)} m_{\lambda}^{(2)}} C\left(j_{\lambda}^{(1)}, j_{\lambda}^{(2)}, J_{\lambda} ; m_{\lambda}^{(1)}, m_{\lambda}^{(2)}, \Omega_{\lambda}\right) \\
& \times Y_{m_{\lambda}^{(1)}}^{j_{\lambda}^{(1)}}\left(\gamma_{\lambda}^{(2,3)}, 0\right) Y_{m_{\lambda}^{(2)}}^{j_{\lambda}^{(2)}}\left(\gamma_{\lambda}^{(1,3)}, \xi_{\lambda}\right) .
\end{aligned}
$$

Using Eqs. (53)-(56) in combination with the definitions of Eqs. (A10)-(A15) we can now compute $\mathcal{X}^{J}$ by means of Eq. (44b), which finally allows us to obtain $\mathbf{R}_{o o}^{J \Pi \Gamma}$ from Eq. (51) and then $\mathbf{S}_{o o}^{J \Pi \Gamma}$ from Eq. (41).

\section{SUMMARY}

A detailed prescription is outlined for calculating stateto-state scattering matrices for tetraatomic systems. The nine-dimensional configuration space is divided into strong interaction, weak interaction, and asymptotic regions. In the strong interaction region, row-orthonormal coordinates ${ }^{2}$ are used which permit a description of the system's wave function in this entire region in terms of single sets of local hyperspherical surface functions at constant values of the hyperradius. This avoids any super-completeness problems. At each hyperradius these functions are arrangement-channelindependent, span all eight hyperangles and are linearly independent, complete, and orthonormal in this hyperangular space. The weak interaction region is divided into different nearly nonoverlapping arrangement channel regions in each of which a different set of arrangement-channel-dependent Delves $^{37,38}$ hyperangular coordinates are used. In both kinds of interaction regions the same principal axes of inertia Euler angles are part of the corresponding coordinate sets. Coupled hyperradial equations are propagated across these regions up to an asymptotic hyperradius where potential energy coupling (but not Coriolis coupling) vanishes. At this boundary a switch to body-fixed Jacobi axes is performed and a detailed prescription is given for projecting, at a constant value of the hyperradius, the scattering wave function onto the separated molecule (diatom-diatom or atom-triatom) wave functions. These two steps (the change in Euler angles and the projec- tion) are done simultaneously. Although they could be done independently of one another, it is more efficient to do them together. This leads to explicit matrix equations relating the logarithmic derivative solutions of the hyperradial equations to the reactance and scattering matrices that are detailed enough to permit a calculation of the latter. Given the favorable kinematic-invariance properties of the row-orthonormal hyperspherical coordinates, the associated ease with which different parity and irreducible representation wave functions can be decoupled, and the ease of parallelization of the related numerical algorithms, this approach should permit benchmark-quality calculations of state-to-state integral and differential cross sections using available massively parallel computers.

A candidate for a first application of this methodology is the $\mathrm{OH}+\mathrm{H}_{2}$ reaction. A preliminary analysis of this system indicates that after a decoupling of the different irreducible representations of the permutation group of three identical atoms and of the two inversion symmetry parities, the dimension $N$ of the square matrices to be propagated is 5000 . We estimate the total time for calculating benchmark-quality state-to-state differential cross sections for this system at 20 total energies between $0.8 \mathrm{eV}$ and $1.2 \mathrm{eV}$ (measured with respect to the bottom of the diatomic potential energy curves for the isolated $\mathrm{H}_{2}$ and $\mathrm{OH}$ molecules) to be approximately $550 \mathrm{~h}$ on a computer having a sustained speed of 100 Gflops for these kinds of calculations, such as the HP V2500 parallel computer at Caltech. Other computers having sustained speeds 30 times or more higher than this are already available, and as a result the $\mathrm{OH}+\mathrm{H}_{2}$ reaction can be studied comfortably using such machines. The feasibility of performing calculations of similar quality for more complicated tetraatomic reactions is determined mainly by the corresponding values of $N$, as the computer time scales as $N^{3}$. The amount of main memory is not expected to be a problem, as it scales as $N^{2}$ only.

\section{ACKNOWLEDGMENTS}

This research was supported in part by NSF Grant No. CHE9810050. One of the authors (K.M.) would like to acknowledge the Carlsberg Foundation for a post doctoral fellowship.

\section{APPENDIX: SPACE-FIXED RADIAL ASYMPTOTIC CONDITIONS}

The asymptotic conditions for the body-fixed $U_{\lambda b_{\lambda}}^{J \Pi \Gamma \lambda^{\prime} b_{\lambda^{\prime}}^{\prime}}\left(r_{\lambda}^{(3)}\right)$ radial functions, defined in Sec. III A, are derived from an analysis of the corresponding space-fixed functions $U_{\lambda u_{\lambda}}^{J \Pi \Gamma \lambda^{\prime} u_{\lambda^{\prime}}^{\prime}}\left(r_{\lambda}^{(3)}\right)$, which is the topic of this appendix. Let us define the space-fixed equivalent of Eq. (30) as

$$
\Psi_{\lambda^{\prime} u_{\lambda^{\prime}}^{\prime}}^{J M \Pi \Gamma} \sim \sum_{\lambda u_{\lambda}} \frac{U_{\lambda u_{\lambda}}^{J \Pi \Gamma \lambda^{\prime} u_{\lambda^{\prime}}^{\prime}}\left(r_{\lambda}^{(3)}\right)}{r_{\lambda}^{(3)}} \Phi_{\lambda u_{\lambda}}^{J M}\left(\mathbf{r}_{\lambda}^{(1)}, \mathbf{r}_{\lambda}^{(2)}, \hat{\mathbf{r}}_{\lambda}^{(3)}\right),
$$

where the space-fixed molecular basis functions of the tetraatomic system, $\Phi_{\lambda u_{\lambda}}^{J M}$, are defined to be simultaneous solu- 
tions of a set of arrangement channel-dependent eigenvalue equations. For diatom-diatom arrangement channels these equations are

$$
\begin{aligned}
& \hat{J}^{2} \Phi_{\lambda u_{\lambda}}^{J M}=\hbar^{2} J(J+1) \Phi_{\lambda u_{\lambda}}^{J M}, \\
& \hat{J}_{\mathrm{sf}_{z}} \Phi_{\lambda u_{\lambda}}^{J M}=\hbar M \Phi_{\lambda u_{\lambda}}^{J M}, \\
& \hat{l}_{\lambda}^{2} \Phi_{\lambda u_{\lambda}}^{J M}=\hbar^{2} l_{\lambda}\left(l_{\lambda}+1\right) \Phi_{\lambda u_{\lambda}}^{J M}, \\
& \hat{J}_{\lambda}^{2} \Phi_{\lambda u_{\lambda}}^{J M}=\hbar^{2} J_{\lambda}\left(J_{\lambda}+1\right) \Phi_{\lambda u_{\lambda}}^{J M}, \\
& {\left[\hat{\mathbf{j}}_{\lambda}^{(i)}\right]^{2} \Phi_{\lambda u_{\lambda}}^{J M}=\hbar^{2} j_{\lambda}^{(i)}\left(j_{\lambda}^{(i)}+1\right) \Phi_{\lambda u_{\lambda}}^{J M}, \quad i=1,2,} \\
& \hat{H}_{\lambda}^{(1,2)} \Phi_{\lambda u_{\lambda}}^{J M}=E_{\lambda \epsilon_{\lambda}} \Phi_{\lambda u_{\lambda}}^{J M},
\end{aligned}
$$

where

$$
\hat{\mathbf{J}}_{\lambda} \equiv \hat{\mathbf{j}}_{\lambda}^{(1)}+\hat{\mathbf{j}}_{\lambda}^{(2)} \text {. }
$$

We have introduced in these equations the compact spacefixed quantum number notation

$$
\lambda u_{\lambda} \equiv\left\{\lambda \epsilon_{\lambda}, J_{\lambda}, l_{\lambda}\right\}=\left\{\lambda_{1}, \lambda_{2}, v_{\lambda}^{(1)}, v_{\lambda}^{(2)}, j_{\lambda}^{(1)}, j_{\lambda}^{(2)}, J_{\lambda}, l_{\lambda}\right\} .
$$

For atom-triatom arrangement channels the set of simultaneous eigenvalue equations are the same as Eq. (A2) except that Eqs. (A2d), (A2e), and (A3) are now substituted by the single expression

$$
\left[\hat{\mathbf{j}}_{\lambda}^{(1)}\right]^{2} \Phi_{\lambda u_{\lambda}}^{J M}=\hbar^{2} j_{\lambda}^{(1)}\left(j_{\lambda}^{(1)}+1\right) \Phi_{\lambda u_{\lambda}}^{J M}
$$

with the corresponding quantum numbers being

$$
\lambda u_{\lambda} \equiv\left\{\lambda \epsilon_{\lambda}, l_{\lambda}\right\}=\left\{\lambda_{1}, \lambda_{2}, \mathbf{v}_{\lambda}^{(1)}, j_{\lambda}^{(1)}, l_{\lambda}\right\} .
$$

Using these definitions, it is straightforward to show that the corresponding radial equations for $U_{\lambda u_{\lambda}}^{J \Pi \Gamma \lambda^{\prime} u_{\lambda^{\prime}}^{\prime}}$ must satisfy the coupled equations

$$
\begin{aligned}
& -\frac{\hbar^{2}}{2 \mu_{\lambda}}\left[\frac{d^{2}}{\left(d r_{\lambda}^{(3)}\right)^{2}}-\frac{l_{\lambda}\left(l_{\lambda}+1\right)}{\left(r_{\lambda}^{(3)}\right)^{2}}\right] U_{\lambda u_{\lambda}}^{J \Pi \Gamma \lambda^{\prime} u_{\lambda^{\prime}}^{\prime}} \\
& +\sum_{\lambda^{\prime \prime} u_{\lambda^{\prime \prime}}^{\prime \prime}}\left\langle\Phi_{\lambda u_{\lambda}}^{J M}\left|V_{\lambda}^{\mathrm{int}}\right| \Phi_{\lambda^{\prime \prime} u_{\lambda^{\prime \prime}}}^{J M}\right\rangle U_{\lambda^{\prime \prime} u_{\lambda^{\prime \prime}}^{\prime \prime}}^{J \Pi \Gamma \lambda^{\prime} u_{\lambda^{\prime}}^{\prime}} \\
& \sim\left(E-E_{\lambda \epsilon_{\lambda}}\right) U_{\lambda u_{\lambda}}^{J \Pi \Gamma \lambda^{\prime} u_{\lambda^{\prime}}^{\prime},}
\end{aligned}
$$

which should be compared to the body-fixed counterpart of Eq. (37). It is important to note that Eq. (A7), as opposed to Eq. (37), does not contain any Coriolis coupling terms, because $\Phi_{\lambda u_{\lambda}}^{J M}$ by definition is an eigenfunctions of the orbital angular momentum operator $\hat{l}_{\lambda}^{2}$. The only coupling term in this equation is the interaction potential coupling which vanishes asymptotically. Consequently, the far asymptotic behavior of the space-fixed radial functions $U_{\lambda u_{\lambda}}^{J \Pi \Gamma \lambda^{\prime} u_{\lambda^{\prime}}^{\prime}}$ is defined from the simple decoupled second order differential equations

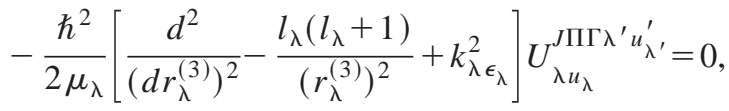

where

$$
k_{\lambda \epsilon_{\lambda}}^{2} \equiv 2 \mu\left(E-E_{\lambda \epsilon_{\lambda}}\right) / \hbar^{2} .
$$

The analytical solutions to this equation, which we will collectively denoted by $X_{\lambda u_{\lambda}}^{J}\left(r_{\lambda}^{(3)}\right)$, can be expressed as

$$
S_{\lambda u_{\lambda}}^{J}\left(r_{\lambda}^{(3)}\right)=\left|k_{\lambda \epsilon_{\lambda}}\right| r_{\lambda}^{(3)}\left\{\begin{array}{l}
y_{l_{\lambda}}\left(k_{\lambda \epsilon_{\lambda}} r_{\lambda}^{(3)}\right) \sin \left(\left(J+J_{\lambda}-l_{\lambda}\right) \pi / 2\right)+j_{l_{\lambda}}\left(k_{\lambda \epsilon_{\lambda}} r_{\lambda}^{(3)}\right) \cos \left(\left(J+J_{\lambda}-l_{\lambda}\right) \pi / 2\right) \quad \text { open } \\
2 i_{l_{\lambda}}\left(\left|k_{\lambda \epsilon_{\lambda}}\right| r_{\lambda}^{(3)}\right) \quad \text { closed, }
\end{array}\right.
$$

and

$$
C_{\lambda u_{\lambda}}^{J}\left(r_{\lambda}^{(3)}\right)=\left|k_{\lambda \epsilon_{\lambda}}\right| r_{\lambda}^{(3)}\left\{\begin{array}{l}
-y_{l_{\lambda}}\left(k_{\lambda \epsilon_{\lambda}} r_{\lambda}^{(3)}\right) \cos \left(\left(J+J_{\lambda}-l_{\lambda}\right) \pi / 2\right)+j_{l_{\lambda}}\left(k_{\lambda \epsilon_{\lambda}} r_{\lambda}^{(3)}\right) \sin \left(\left(J+J_{\lambda}-l_{\lambda}\right) \pi / 2\right) \\
2 k_{l_{\lambda}}\left(\left|k_{\lambda \epsilon_{\lambda}}\right| r_{\lambda}^{(3)}\right) \quad \text { open }
\end{array}\right.
$$

for the real standing wave formulation, and

$$
I_{\lambda u_{\lambda}}^{J}\left(r_{\lambda}^{(3)}\right)=\left|k_{\lambda \epsilon_{\lambda}}\right| r_{\lambda}^{(3)}\left\{\begin{array}{l}
\exp \left[+i\left(J+J_{\lambda}-l_{\lambda}\right) \pi / 2\right] h_{l_{\lambda}}^{(-)}\left(k_{\lambda \epsilon_{\lambda}} r_{\lambda}^{(3)}\right) \text { open } \\
2 i_{l_{\lambda}}\left(\left|k_{\lambda \epsilon_{\lambda}}\right| r_{\lambda}^{(3)}\right) \quad \text { closed, }
\end{array}\right.
$$

and

$$
O_{\lambda u_{\lambda}}^{J}\left(r_{\lambda}^{(3)}\right)=\left|k_{\lambda \epsilon_{\lambda}}\right| r_{\lambda}^{(3)}\left\{\begin{array}{l}
\exp \left[-i\left(J+J_{\lambda}-l_{\lambda}\right) \pi / 2\right] h_{l_{\lambda}}^{(+)}\left(k_{\lambda \epsilon_{\lambda}} r_{\lambda}^{(3)}\right) \quad \text { open } \\
2 k_{l_{\lambda}}\left(\left|k_{\lambda \epsilon_{\lambda}}\right| r_{\lambda}^{(3)}\right) \quad \text { closed }
\end{array}\right.
$$


for the complex propagating spherical wave formulation. The open and closed cases for each of the four functions refer to channels that are energetically open $\left(E \geqslant E_{\lambda \epsilon_{\lambda}}\right)$ or closed $\left(E<E_{\lambda \epsilon_{\lambda}}\right)$, i.e., when the linear momentum $k_{\lambda \epsilon_{\lambda}}$ defined from Eq. (A9) is respectively real or pure positive imaginary. $h_{\lambda}^{( \pm)}=-y_{l_{\lambda}} \pm i j_{l_{\lambda}}, y_{l_{\lambda}}, j_{l_{\lambda}}, i_{l_{\lambda}}$, and $k_{l_{\lambda}}$ are the spherical Bessel functions of Ref. 42, page 435. The angle $\left(J+J_{\lambda}\right.$ $\left.-l_{\lambda}\right) \pi / 2$ appearing in these equations is an arbitrary but convenient phase.

The asymptotic behavior of the body-fixed counterpart of Eq. (A8) is finally obtained through the transformation relation

$$
\begin{aligned}
\Phi_{\lambda b_{\lambda}}^{J M}\left(\mathbf{r}_{\lambda}^{(1)}, \mathbf{r}_{\lambda}^{(2)}, \hat{\mathbf{r}}_{\lambda}^{(3)}\right) \\
=(-1)^{J_{\lambda}-\Omega_{\lambda}} \sum_{l_{\lambda}} C\left(J, J_{\lambda}, l_{\lambda} ; \Omega_{\lambda},-\Omega_{\lambda}, 0\right) \\
\quad \times \Phi_{\lambda u_{\lambda}}^{J M}\left(\mathbf{r}_{\lambda}^{(1)}, \mathbf{r}_{\lambda}^{(2)}, \hat{\mathbf{r}}_{\lambda}^{(3)}\right),
\end{aligned}
$$

where the $C$ 's are the usual Clebsch-Gordan coupling coefficients and $(-1)^{J_{\lambda}-\Omega_{\lambda}}$ is a convenient phase factor. This relation finally leads to the important similarity transformation

$$
\begin{aligned}
\mathcal{X}_{\lambda b_{\lambda}}^{J \lambda^{\prime \prime} \delta_{\lambda^{\prime \prime}}^{\prime \prime}\left(r_{\lambda}^{(3)}\right)=} & \delta_{\lambda}^{\lambda^{\prime \prime}}(-1)^{\Omega_{\lambda}-\Omega_{\lambda}^{\prime \prime}} \sum_{l_{\lambda}} C\left(J, J_{\lambda}, l_{\lambda} ; \Omega_{\lambda},-\Omega_{\lambda}, 0\right) \\
& \times C\left(J, J_{\lambda}, l_{\lambda} ; \Omega_{\lambda}^{\prime \prime}-\Omega_{\lambda}^{\prime \prime}, 0\right) X_{\lambda u_{\lambda}}^{J}\left(r_{\lambda}^{(3)}\right)
\end{aligned}
$$

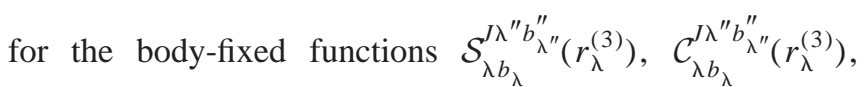
$\mathcal{I}_{\lambda b_{\lambda}}^{J \lambda^{\prime \prime} b^{\prime \prime}}\left(r_{\lambda}^{(3)}\right)$, and $\mathcal{O}_{\lambda b_{\lambda}}^{J \lambda^{\prime \prime} b^{\prime \prime}}\left(r_{\lambda}^{(3)}\right)$ entering Eq. (39). Thus in Eq. (A15) $\{\mathcal{X}, X\}$ can be any of the sets $\{\mathcal{S}, S\},\{\mathcal{C}, C\},\{\mathcal{I}, I\}$, or $\{\mathcal{O}, O\}$.

${ }^{1}$ A. S. Davydov, Quantum Mechanics, 2nd edition (Pergamon Press, Oxford, 1976), p. 169.

${ }^{2}$ A. Kuppermann, J. Phys. Chem. 101, 6368 (1997).

${ }^{3}$ A. Kuppermann, Chem. Phys. Lett. 132, 374 (1975).

${ }^{4}$ J. M. Launay and M. le Dourneuf, Chem. Phys. Lett. 163, 179 (1989).

${ }^{5}$ J. M. Launay and M. le Dourneuf, Chem. Phys. Lett. 169, 473 (1990).

${ }^{6}$ J. M. Launay, Theor. Chim. Acta 78, 183 (1991).

${ }^{7}$ J. M. Launay and S. B. Padkjaer, Chem. Phys. Lett. 181, 95 (1991).

${ }^{8}$ S. Branchett, S. B. Padkjaer, and J. M. Launay, Chem. Phys. Lett. 208, $523(1993)$
${ }^{9}$ Y.-S. M. Wu, A. Kuppermann, and B. Lepetit, Chem. Phys. Lett. 186, 319 (1991).

${ }^{10}$ Y.-S. M. Wu and A. Kuppermann, Chem. Phys. Lett. 235, 105 (1993).

${ }^{11}$ A. Kuppermann and Y.-S. M. Wu, Chem. Phys. Lett. 205, 577 (1993).

${ }^{12}$ A. Kuppermann and Y.-S. M. Wu, Chem. Phys. Lett. 213, 636 (1993)

${ }^{13}$ B. Lepetit, Z. Peng, and A. Kuppermann, Chem. Phys. Lett. 166, 572 (1990).

${ }^{14}$ B. Lepetit and A. Kuppermann, Chem. Phys. Lett. 166, 581 (1990).

${ }^{15}$ Y.-S. M. Wu and A. Kuppermann, Chem. Phys. Lett. 235, 105 (1995).

${ }^{16}$ A. Kuppermann and Y.-S. M. Wu, Chem. Phys. Lett. 241, 229 (1995).

${ }^{17}$ R. T. Pack and G. A. Parker, J. Chem. Phys. 87, 3888 (1987).

${ }^{18}$ R. T. Pack and G. A. Parker, J. Chem. Phys. 90, 3511 (1989).

${ }^{19}$ J. D. Kress, Z. Bacic, G. A. Parker, and R. T Pack, Chem. Phys. Lett. 157, 484 (1989).

${ }^{20}$ Z. Bacic, J. D. Kress, G. A. Parker, and R. T Pack, J. Chem. Phys. 92, 2344 (1990)

${ }^{21}$ J. D. Kress, R. B. Walker, and E. F. Hayes, J. Chem. Phys. 93, 8085 (1990).

${ }^{22}$ Z. Darakjan, E. F. Hayes, G. A. Parker, E. A. Butcher, and J. D. Kress, J. Chem. Phys. 95, 2516 (1991).

${ }^{23}$ G. A. Parker and R. T. Pack, J. Chem. Phys. 98, 6883 (1993).

${ }^{24}$ R. T. Pack, E. A. Butcher, and G. A. Parker, J. Chem. Phys. 99, 9310 (1993)

${ }^{25}$ J. Linderberg, S. B. Padkjaer, Y. Ohrn, and B. Vessal, J. Chem. Phys. 90, 6254 (1989).

${ }^{26}$ A. Kuppermann and P. G. Hipes, J. Chem. Phys. 84, 5962 (1986).

${ }^{27}$ P. G. Hipes and A. Kuppermann, Chem. Phys. Lett. 133, 1 (1987).

${ }^{28}$ S. A. Cuccaro, P. G. Hipes, and A. Kuppermann, Chem. Phys. Lett. 154, 155 (1989).

${ }^{29}$ Y.-S. M. Wu, S. A. Cuccaro, P. G. Hipes, and A. Kuppermann, Chem. Phys. Lett. 168, 429 (1990).

${ }^{30}$ Y.-S. M. Wu, S. A. Cuccaro, P. G. Hipes, and A. Kuppermann, Theor. Chim. Acta 79, 225 (1991).

${ }^{31}$ G. C. Schatz, Chem. Phys. Lett. 150, 92 (1988).

${ }^{32}$ G. C. Schatz, Chem. Phys. Lett. 151, 409 (1988).

${ }^{33}$ S. Pogrebnya, J. Palma, D. C. Clary, and J. Echave, Phys. Chem. Chem. Phys. 2, 693 (2000)

${ }^{34}$ J. Kaye and A. Kuppermann, Chem. Phys. Lett. 78, 546 (1981).

${ }^{35}$ A. Kuppermann, in Advances in Molecular Vibrations and Collision Dynamics, edited by J. M. Bowman (JAI, Greenwich, 1993), Vol. 2B, pp. $117-186$.

${ }^{36}$ M. Hamermesh, Group Theory (Addison-Wesley, Reading, MA, 1962), Chap. 7.

${ }^{37}$ L. M. Delves, Nucl. Phys. 9, 39 (1959).

${ }^{38}$ L. M. Delves, Nucl. Phys. 20, 275 (1960).

${ }^{39}$ G. C. Schatz and A. Kuppermann, J. Chem. Phys. 11, 4642 (1976).

${ }^{40}$ A. M. Lane and R. G. Thomas, Rev. Mod. Phys. 30, 257 (1958).

${ }^{41}$ S. F. Mott and H. S. W. Massey, The Theory of Atomic Collisions (Clarendon, Oxford, UK, 1956), Chaps. 14 and 15.

${ }^{42}$ M. Abramowitz and I. A. Stegun, Handbook of Mathematical Functions (Dover, New York, 1968). 